\title{
Space Launch System Booster Separation Aerodynamic Database Development and Uncertainty Quantification
}

\author{
David T. Chan* \\ Jeremy T. Pinier ${ }^{\dagger}$, Floyd J. Wilcox, Jr. ${ }^{\ddagger}$ \\ NASA Langley Research Center, Hampton, VA, 23681 \\ Derek J. Dalle ${ }^{\S}$, Stuart E. Rogers $₫$ \\ NASA Ames Research Center, Moffett Field, CA, 94035 \\ Reynaldo J. Gomez ${ }^{\|}$ \\ NASA Johnson Space Center, Houston, TX, 77058
}

\begin{abstract}
The development of the aerodynamic database for the Space Launch System (SLS) booster separation environment has presented many challenges because of the complex physics of the flow around three independent bodies due to proximity effects and jet interactions from the booster separation motors and the core stage engines. This aerodynamic environment is difficult to simulate in a wind tunnel experiment and also difficult to simulate with computational fluid dynamics. The database is further complicated by the high dimensionality of the independent variable space, which includes the orientation of the core stage, the relative positions and orientations of the solid rocket boosters, and the thrust levels of the various engines. Moreover, the clearance between the core stage and the boosters during the separation event is sensitive to the aerodynamic uncertainties of the database. This paper will present the development process for Version 3 of the SLS booster separation aerodynamic database and the statistics-based uncertainty quantification process for the database.
\end{abstract}

\section{Nomenclature}

$\begin{array}{llll}\text { Symbols } & & k & \text { Range Analysis coverage factor } \\ C A & \text { Axial force coefficient, body axis } & M_{\infty} & \text { Freestream Mach number } \\ C L L & \text { Rolling moment coefficient, body axis } & q_{\infty} & \text { Margin Index } \\ C L M & \text { Pitching moment coefficient, body } & R & \text { Freestream dynamic pressure, psf } \\ & \text { axis } & R / d_{2} & \text { Range (maximum - minimum) } \\ C L N & \text { Yawing moment coefficient, body } & U M F & \text { Uncertainty Model Factor } \\ & \text { axis } & V_{\infty} & \text { Freestream velocity, ft/sec } \\ C N & \text { Normal force coefficient, body axis } & \alpha & \text { Angle of Attack, deg } \\ C T & \text { Thrust coefficient } & \beta & \text { Angle of Sideslip, deg } \\ C Y & \text { Side force coefficient, body axis } & \Delta X & \text { Position of SRB nose relative to }\end{array}$

*Research Aerospace Engineer. Configuration Aerodynamics Branch. Member AIAA. david.t.chan@nasa.gov

${ }^{\dagger}$ Research Aerospace Engineer. Configuration Aerodynamics Branch. Associate Fellow AIAA. jeremy.t.pinier@nasa.gov

${ }^{\ddagger}$ Research Aerospace Engineer. Configuration Aerodynamics Branch. Member AIAA. floyd.j.wilcox@nasa.gov

$\S$ Research Scientist/Engineer. Computational Aerosciences Branch. Member AIAA. derek.j.dalle@nasa.gov

ฯAerospace Engineer. Computational Aerosciences Branch. Associate Fellow AIAA. stuart.e.rogers@nasa.gov

" Aerospace Engineer. Applied Aerosciences and CFD Branch. Member AIAA. reynaldo.j.gomez@nasa.gov 


\begin{tabular}{|c|c|c|c|}
\hline & mated position in $\mathrm{x}$-direction, $\mathrm{ft}$ & CSE & Core Stage Engine \\
\hline$\Delta Y$ & $\begin{array}{l}\text { Position of SRB nose relative to } \\
\text { mated position in y-direction, } \mathrm{ft}\end{array}$ & DACE & $\begin{array}{l}\text { Design and Analysis of Computer } \\
\text { Experiments }\end{array}$ \\
\hline \multirow[t]{2}{*}{$\Delta Z$} & Position of SRB nose relative to & DB & Database \\
\hline & mated position in z-direction, $\mathrm{ft}$ & DOF & Degrees of Freedom \\
\hline \multirow[t]{2}{*}{$\Delta \phi$} & Roll rotation angle from core body & $\mathrm{F} \& \mathrm{M}$ & Force \& Moment \\
\hline & axis to SRB body axis, deg & GN\&C & Guidance, Navigation, and Control \\
\hline \multirow[t]{2}{*}{$\Delta \psi$} & Yaw rotation angle from core body & LAS & Launch Abort System \\
\hline & axis to SRB body axis, deg & $\mathrm{LH}$ & Left Hand \\
\hline$\Delta \theta$ & $\begin{array}{l}\text { Pitch rotation angle from core body } \\
\text { axis to SRB body axis, deg }\end{array}$ & $\begin{array}{l}\text { LSRB } \\
\text { MPCV }\end{array}$ & $\begin{array}{l}\text { Left (Port) Solid Rocket Booster } \\
\text { Multi-Purpose Crew Vehicle }\end{array}$ \\
\hline$\sigma$ & Standard deviation & MRP & Moment Reference Point \\
\hline \multicolumn{2}{|l|}{ Units } & NASA & $\begin{array}{l}\text { National Aeronautics and Space } \\
\text { Administration }\end{array}$ \\
\hline$\stackrel{\circ}{\circ} \operatorname{deg}$ & degrees & OVERFLOW & OVERset grid CFD FLOW solver \\
\hline & & $\mathrm{RH}$ & Right Hand \\
\hline & $\begin{array}{l}\text { pounds per square foot } \\
\text { second }\end{array}$ & RSRB & $\begin{array}{l}\text { Right (Starboard) Solid Rocket } \\
\text { Booster }\end{array}$ \\
\hline \multicolumn{2}{|l|}{ Acronyms } & SLS & Space Launch System \\
\hline BSM & Booster Separation Motor & SPC & Statistical Process Control \\
\hline \multirow[t]{2}{*}{ Cart3D } & Cartesian grid 3-Dimensional inviscid & SRB & Solid Rocket Booster \\
\hline & CFD analysis package & UPWT & Unitary Plan Wind Tunnel \\
\hline CFD & Computational Fluid Dynamics & UQ & Uncertainty Quantification \\
\hline CG & Center of Gravity & WT & Wind Tunnel \\
\hline
\end{tabular}

\section{Introduction}

$T^{\text {HE }}$ Space Launch System (SLS) ${ }^{1}$ is NASA's advanced heavy-lift launch vehicle that will enable human 1 exploration beyond Earth's orbit for the United States. The SLS is designed to launch astronaut crews aboard the Orion Multi-Purpose Crew Vehicle (MPCV) on missions to explore multiple, deep-space destinations. The SLS design will continue to evolve into configurations with increasingly higher lift capacity and the capability for both crew and cargo missions as shown in Figure 1. The aerodynamic characterization of the initial Block 1 configuration has been the focus of the SLS program in recent years and the development of Version 3 of the Booster Separation Aerodynamic Database is the focus of this paper.

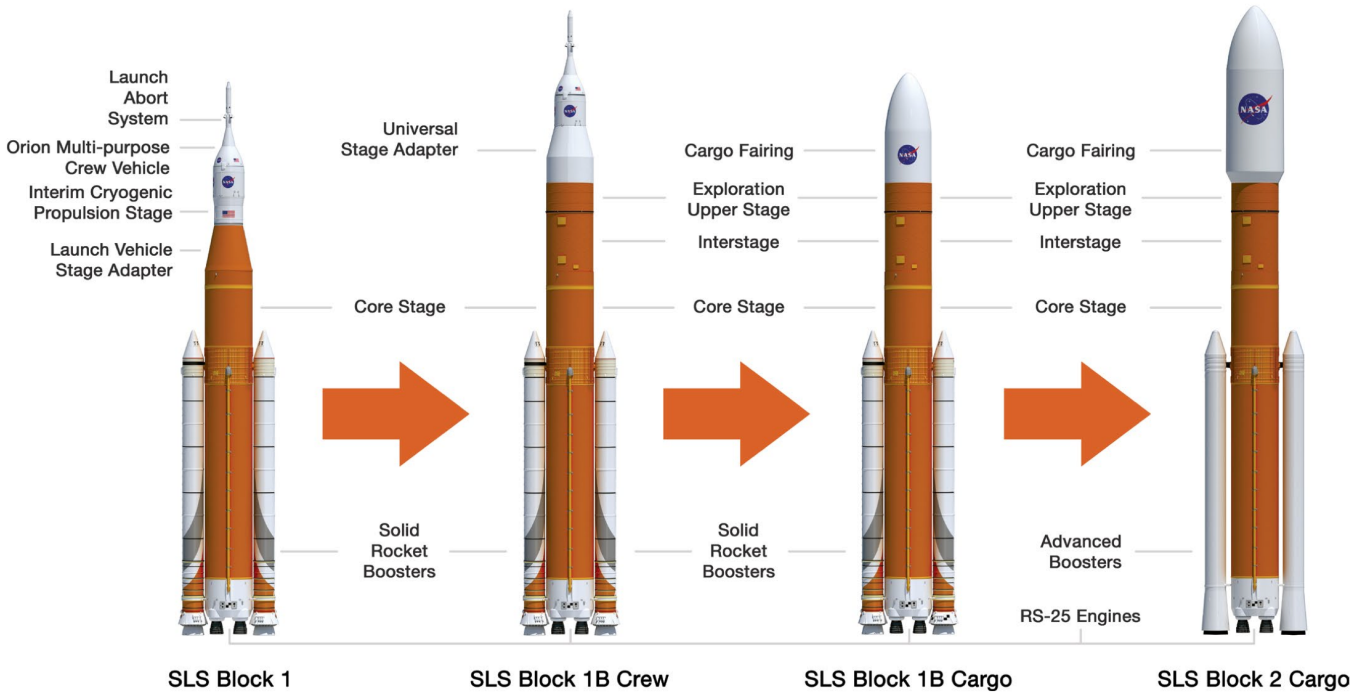

Figure 1. Space Launch System planned configurations for crew and cargo missions. Graphic obtained from www.nasa.gov/sls. 
The SLS Block 1 launch vehicle consists of a central core stage that is powered by four RS-25 liquid propellent core stage engines (CSE) and by two five-segment solid rocket boosters (SRB) attached to each side of the core stage (Figure 2). At the completion of the main boost phase of ascent when the SRBs have nearly consumed their fuel supply, the SRBs are separated from the core stage and expended. During this booster separation event, sixteen total booster separation motors (BSM), eight on each SRB mounted in clusters of four near the front and rear of each SRB, fire for a short duration to move the SRBs safely away from the core stage (Figure $3)$.

During the initial portion of the booster separation event, it is very important to maintain a safe clearance between the SRBs and the core stage to ensure that the SRBs will not re-contact the core stage. The clearance distances are small and can be affected by the complex flow physics at high supersonic Mach numbers around the three independent bodies (core and 2 SRBs), therefore characterization of the aerodynamics of each body is of high importance. In SLS Guidance, Navigation, and Control (GN\&C) simulations of previous iterations of the booster separation aerodynamic database, it was determined that the likelihood of re-contact between the boosters and core stage was very sensitive to the aerodynamic uncertainties of the SRBs.

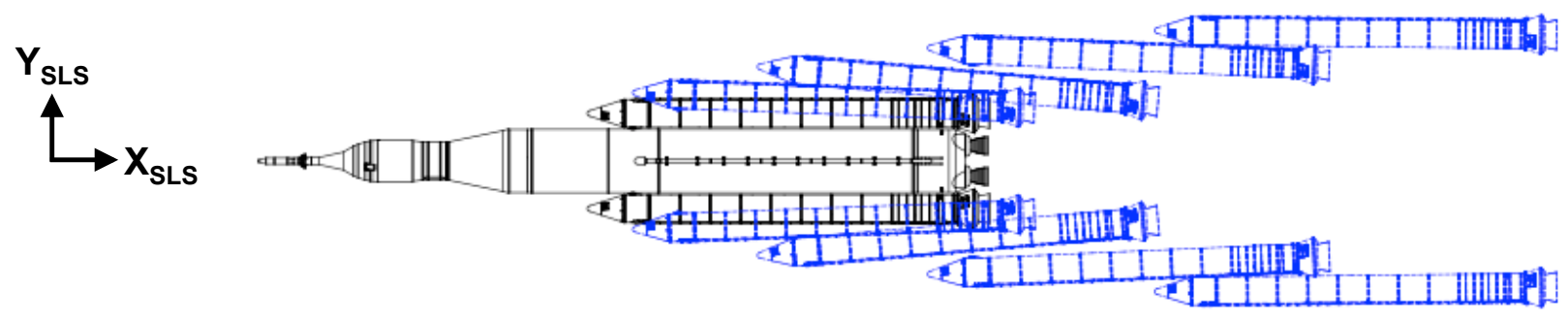

(a) Top View (X-Y plane)

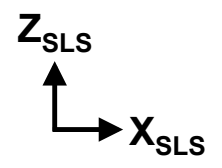

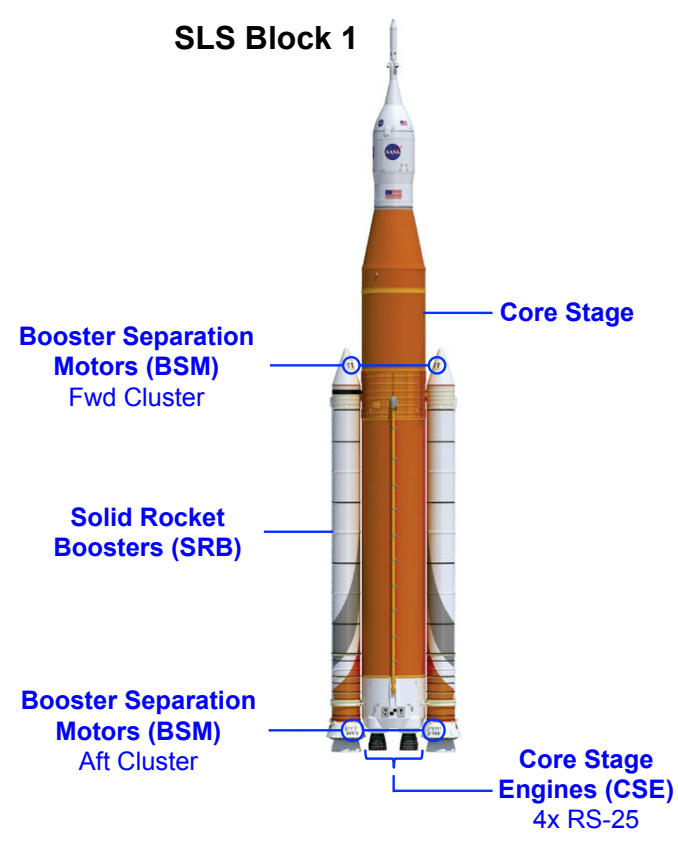

Figure 2. Main components of the SLS launch vehicle during the booster separation event.

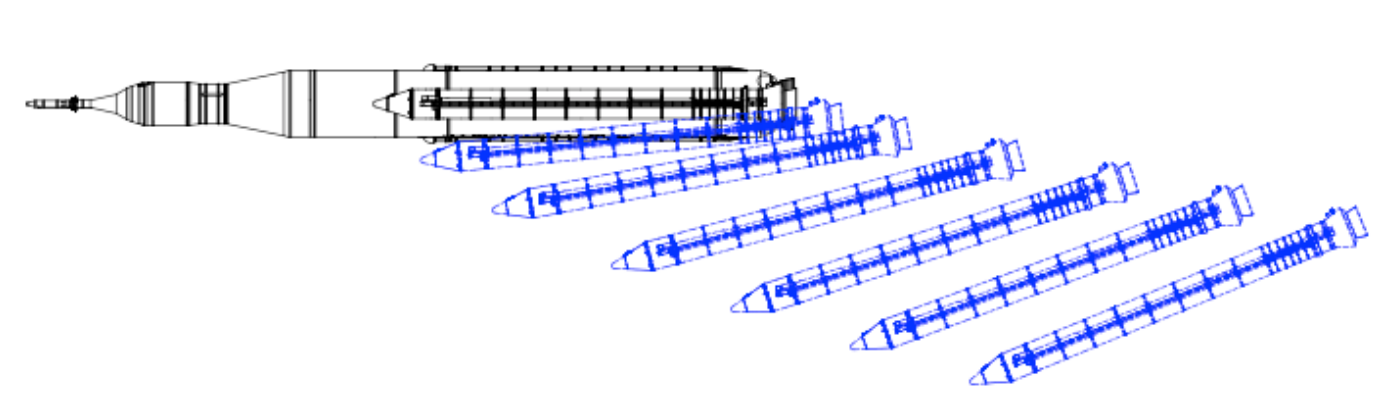

(b) Side View (X-Z plane)

Figure 3. Diagram of nominal SLS booster separation event shown from the (a) top view and (b) side view.

However, this aerodynamic environment is difficult to simulate in either a wind tunnel (WT) experiment or in computational fluid dynamics (CFD) due to proximity effects between the three separating bodies and jet-induced effects from the twenty-two total engine plumes between the BSMs, SRBs, and CSEs. In particular, the front BSMs fire in a partially forward upstream direction even causing plume impingement on the core stage, and the plumes are highly under-expanded due to the low dynamic pressure at high altitude 
of the booster separation event. The highly under-expanded plumes affect a large volume of flow and cause complex interactions with the oncoming high-speed freestream flow.

Furthermore, development of the booster separation aerodynamic database is complicated by the high dimensionality of the independent variable space. In order to properly characterize the aerodynamics, the orientation of the core stage, the location and orientation of the SRBs in relation to the core, the thrust levels of the multiple engines, and the freestream flow conditions, are all required information. Simplifying assumptions were made to reduce the number of independent parameters to allow the booster separation aerodynamic environment to be characterized in CFD solutions and a WT test.

The development and uncertainty quantification process for Version 3 of the SLS Block 1 Booster Separation Aerodynamic Force and Moment (F\&M) Database will be presented in this paper. The nominal values are developed using a large set of CFD solutions, while a mix of wind tunnel data and CFD solutions are used to help quantify the aerodynamic uncertainties. The goal of Version 3 of the database was to improve the aerodynamic characterization of the booster separation event over previous iterations of the database and to reduce the aerodynamic uncertainties associated with the database, especially in the near-proximity region during the initial portion of the booster separation event.

\section{Aerodynamic Data Sources}

The original plan for Version 3 of the SLS Booster Separation aerodynamic database was to conduct a large wind tunnel test campaign that would cover the entire parameter space and form the basis of the database, similar to the booster separation wind tunnel tests conducted in the 1970s and 1980s for the NASA Space Shuttle program. The wind tunnel test would use high pressure air to simulate the BSM engine plumes, but the SRB and CSE engine plumes would not be simulated. However, due to program resource constraints, the decision was made to instead use a large number of relatively inexpensive Cart3D CFD solutions to build the database. Cart3D is an inviscid CFD analysis package developed by NASA that performs automated CFD analysis on complex geometry, supports steady and time-dependent simulations, and features fullyintegrated adjoint-driven mesh adaptation. ${ }^{2}$ In addition to the cost benefit, the Cart3D CFD solutions would be able to simulate all of the engine plumes from the BSMs, SRBs, and CSEs concurrently and capture any plume impingement on any of the three bodies.

The major risk with using inviscid Cart3D CFD solutions to build the database is trusting that the complex flow physics present during the booster separation event are adequately captured in the solutions. As part of the risk reduction effort, a limited set of high-fidelity viscous CFD solutions using OVERFLOW were also acquired to provide a comparison data set. OVERFLOW is a three-dimensional time-marching implicit Navier-Stokes CFD code developed by NASA that uses structured overset grid systems, has options for thin layer or full viscous terms, and allows algebraic, one-equation, or two-equation turbulence models. ${ }^{3}$ Although it would be preferable to use solutions from a high-fidelity CFD flow solver such as OVERFLOW to build the database, the complexity and scope of the booster separation aerodynamic environment prevented this option due to time and resource constraints. However, the acquired OVERFLOW data set proved invaluable in helping to improve the quality of the Cart3D CFD solutions by providing insights into features of the complex flow structure that led to an improvement in the Cart3D output-based grid adaptation methodology.

Figure 4 shows example flow field pressure contours from a representative near-proximity booster separation solution from both Cart3D and OVERFLOW. It should be noted that these are representative examples only and are not meant for direct comparison of flow fields since the two cases are not at the same separation distances nor are the color scales the same. However, the main flow field feature to note is how the plumes from the partially forward-firing BSMs of the front cluster significantly effect the shock structure from the front of the vehicle and cause a plume-induced pressure rise on the service module of the MPCV. The BSM plumes also impinge on the core stage in the near-proximity separation region and as the boosters continue to move away from the core stage in the longitudinal direction, the CSE plumes also impinge on the aft end of the SRBs. Details of the Cart3D and OVERFLOW booster separation CFD solution methodology will not be discussed in this paper, but more information can be found in Refs. 4, 5, and 6 .

Furthermore, a reduced-scope wind tunnel test was conducted on a 0.9\%-scale SLS booster separation model in the NASA Langley Research Center (LaRC) Unitary Plan Wind Tunnel (UPWT) that concentrated on the near-proximity and mid-proximity axial separation distances between the core stage and the SRBs. The SRBs were mounted to a separation rig that allowed the SRBs to be positioned in the desired location 


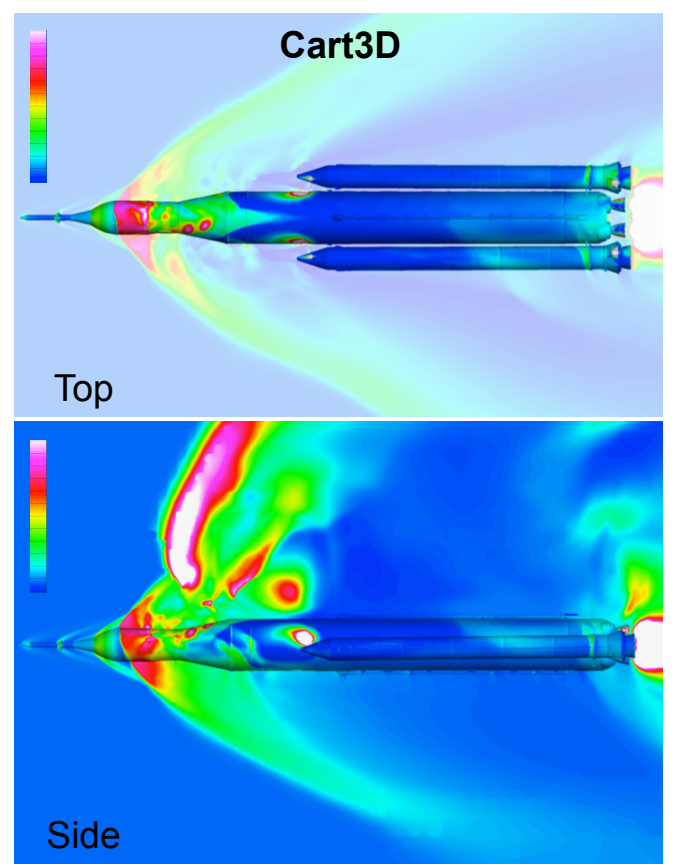

(a) Cart3D
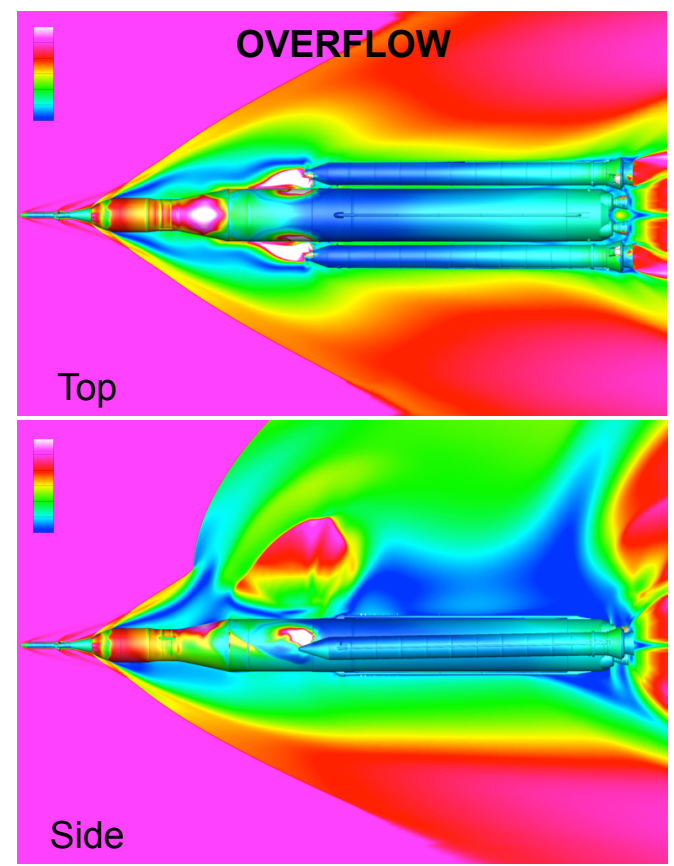

(b) OVERFLOW

Figure 4. Example flow field pressure contours for booster separation near-proximity CFD solution from (a) Cart3D and (b) OVERFLOW. Note : Representative examples only; not meant for direct comparison of flow fields.

and orientation relative to the core and also allowed plume simulation of the BSMs through the use of high pressure air. A photograph of the wind tunnel model is shown in Figure 5. Further details can be found in Ref. 7. A large set of Cart3D CFD solutions were computed on the WT model configuration at similar separation parameter break points and flow conditions as acquired in the UPWT. This Cart3D data set covered the entire wind tunnel test matrix for the nominal model configuration with all BSMs firing. The comparisons between this Cart3D data set and the wind tunnel data set also helped with refinement of the Cart3D grid adaptation methodology.

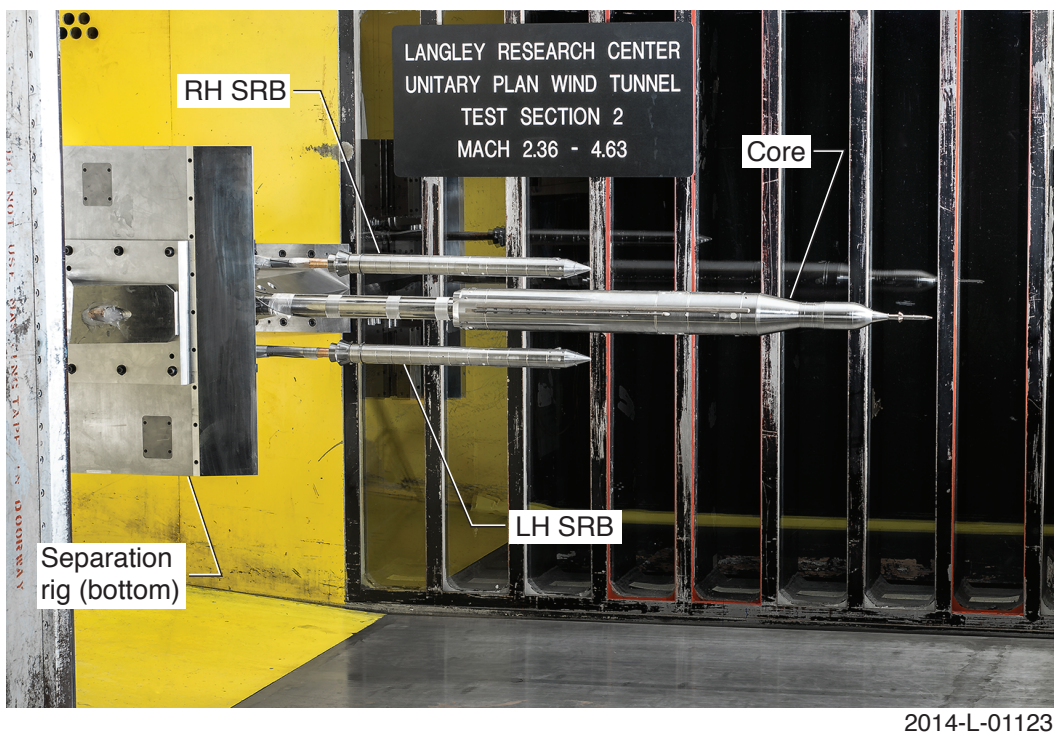

Figure 5. Photograph of the 0.9\%-scale SLS Booster Separation wind tunnel model in the NASA Langley Unitary Plan Wind Tunnel. 
The three main sources of data that were acquired to characterize the aerodynamics of the core and SRBs during the booster separation event (inviscid Cart3D CFD solutions, viscous OVERFLOW CFD solutions, and wind tunnel data from the NASA LaRC UPWT) were divided between the flight (full-scale) configuration of the SLS and the wind tunnel $(0.9 \%$-scale) configuration used in the UPWT. These data sets were used in different ways in the development of the aerodynamic database, as the Cart3D data set of the flight configuration were used primarily for developing and constructing the aerodynamic database nominal values, while all of the data sets were used for quantifying the aerodynamic uncertainties associated with the database. This is summarized graphically in Figure 6 .

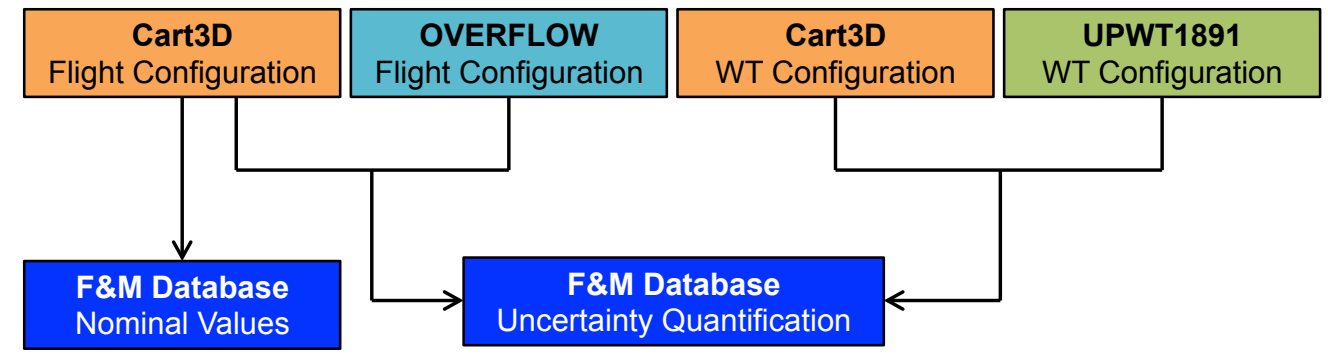

Figure 6. CFD and WT data source usage for Booster Separation aerodynamic database.

Most of the data sets acquired were focused on the baseline flight configuration where the BSMs, SRBs, and CSEs are all operating nominally. However, an off-nominal database was also required for the unlikely case that one of the CSEs fails during ascent. Figure 7 shows the numbering scheme for the CSEs. The engines are numbered in a counter-clockwise order starting with the top left engine, therefore CSE \#1 \& CSE \#2 are on the left side of the core stage and CSE \#3 \& CSE \#4 are on the right side of the core stage. Data sets were acquired for the scenario where CSE \#3 fails and separate data sets were acquired for the scenario where CSE \#4 fails. It was determined that the aerodynamic effect was significantly different between these two scenarios to warrant separate data sets. Symmetry assumptions are used in the aerodynamic database to handle the CSE \#1 and CSE \#2 failure scenarios. A plane of symmetry is assumed to exist between the left and right sides of the vehicle, therefore data from one side can be reflected to the other side with the appropriate transformation equations.

Furthermore, there were separate Cart3D data sets of the flight configuration that were used to evaluate database modeling decisions, such as symmetry assumptions and database interpolation, and these data sets were used in the database uncertainty quantification process. A summary of how all of the data sets were used in the development of the aerodynamic database is shown in Table 1.

All of the aerodynamic force and moment coefficients from the various data sets are forebody coefficients meaning that the base forces are not included for each of the three bodies. Furthermore, the static thrust contributions from each BSM, SRB, and CSE are removed from the force and moment data. In the CFD solutions, this meant that for the core

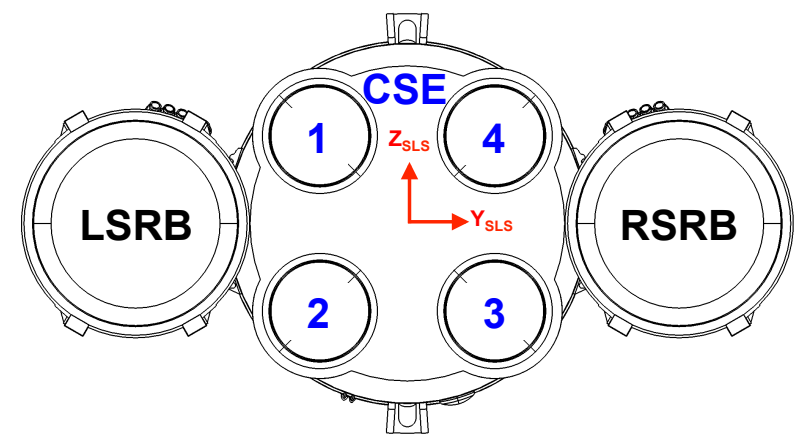

Figure 7. Numbering scheme for the core stage engines (CSE). stage, all surfaces except for the CSE surfaces and the core base were used for the integrated F\&M coefficients and the CSE static thrust was not included. Similarly for the SRBs, all surfaces except for the SRB engine surfaces, the SRB base, and the internal BSM nozzle surface, were used for the integrated F\&M coefficients and the BSM and SRB static thrust were not included. In the UPWT data, a base force correction was applied to the core stage F\&M data using base pressure data and the BSM static thrust was removed from the SRB F\&M data. The UPWT test did not simulate the CSE or SRB engine plumes. 
Table 1. Summary of booster separation data sets and their usage in the development of the aerodynamic database.

\begin{tabular}{|c|c|c|c|c|c|c|c|}
\hline $\begin{array}{c}\text { Data } \\
\text { Set }\end{array}$ & Purpose & $\begin{array}{c}\text { Database } \\
\text { Usage }\end{array}$ & Source & Config & BSM & CSE & $\begin{array}{c}\text { Data } \\
\text { Points } \\
\end{array}$ \\
\hline \multirow{3}{*}{1} & \multirow{3}{*}{$\begin{array}{c}\text { Build DB } \\
\text { nominal } \\
\text { values } \\
\end{array}$} & \multirow{3}{*}{ Nominals } & \multirow{3}{*}{ Cart3D } & \multirow{3}{*}{ Flight } & \multirow{3}{*}{ Nominal } & Nominal & 9,306 \\
\hline & & & & & & \#3 out & 6,358 \\
\hline & & & & & & \#4 out & 6,358 \\
\hline 2 & $\begin{array}{l}\text { Evaluate effect } \\
\text { of one-BSM-out }\end{array}$ & Uncertainty & Cart3D & Flight & One-BSM-out & Nominal & 75 \\
\hline \multirow{3}{*}{3} & \multirow{3}{*}{$\begin{array}{c}\text { Evaluate database } \\
\text { interpolation } \\
\text { error }\end{array}$} & \multirow{3}{*}{ Uncertainty } & \multirow{3}{*}{ Cart3D } & \multirow{3}{*}{ Flight } & \multirow{3}{*}{ Nominal } & Nominal & 54 \\
\hline & & & & & & \#3 out & 50 \\
\hline & & & & & & \#4 out & 50 \\
\hline 4 & $\begin{array}{c}\text { Evaluate beta and } \\
\text { RH/LH SRB } \\
\text { symmetry }\end{array}$ & Uncertainty & Cart3D & Flight & Nominal & Nominal & 9,306 \\
\hline \multirow{2}{*}{5} & \multirow{2}{*}{$\begin{array}{l}\text { Evaluate CSE } \\
\text { symmetry }\end{array}$} & \multirow{2}{*}{ Uncertainty } & \multirow{2}{*}{ Cart3D } & \multirow{2}{*}{ Flight } & \multirow{2}{*}{ Nominal } & $\# 3$ out & 8 \\
\hline & & & & & & \#4 out & 8 \\
\hline \multirow{3}{*}{6} & \multirow{3}{*}{$\begin{array}{c}\text { Evaluate Cart3D } \\
\text { against high- } \\
\text { fidelity CFD } \\
\end{array}$} & \multirow{3}{*}{ Uncertainty } & \multirow{3}{*}{ OVERFLOW } & \multirow{3}{*}{ Flight } & \multirow{3}{*}{ Nominal } & Nominal & 232 \\
\hline & & & & & & \#3 out & 82 \\
\hline & & & & & & \#4 out & 82 \\
\hline \multirow{2}{*}{7} & \multirow{2}{*}{$\begin{array}{l}\text { Evaluate Cart3D } \\
\text { against WT data }\end{array}$} & \multirow{2}{*}{ Uncertainty } & Cart3D & \multirow{2}{*}{ WT } & \multirow{2}{*}{ Nominal } & \multirow{2}{*}{$\mathrm{N} / \mathrm{A}$} & 6,316 \\
\hline & & & UPWT & & & & $>10^{5}$ \\
\hline
\end{tabular}

\section{Aerodynamic Database Development}

Version 3 of the SLS Booster Separation Aerodynamic Force and Moment Database provides the six degree of freedom (DOF) total aerodynamic force and moment coefficients for each of the three bodies (core stage and two SRBs) during the booster separation event. The total aerodynamic coefficients provided in the database include the combined aerodynamic effect of proximity among the three separating bodies and jet plume interactions from all of the engine plumes.

The booster separation database covers the nominal operation of the BSMs and CSEs, but also covers offnominal engine operation scenarios such as a one-CSE-out or a one-BSM-out failure. For the one-BSM-out scenario, the aerodynamics looked very similar to the nominal BSM operation scenario, so a separate database was not developed because it was deemed unnecessary. This is because the loss of one BSM in a cluster of four did not change the size and effect of the overall BSM plumes since the plumes are all highly underexpanded. However, separate uncertainty tables were developed for the one-BSM-out scenario to account for this database modeling decision. In summary, the database consists of the following components:

1. 6-DOF total aerodynamic force and moment coefficients for the Core and RSRB for nominal BSM and CSE operation.

- The LSRB data are obtained through left-right symmetry assumptions and equations.

- The data for the one-BSM-out scenario are also obtained using this data set.

2. 6-DOF total aerodynamic force and moment coefficients for the Core, RSRB, and LSRB for nominal BSM operation, but CSE \#3 inoperable.

- The data for the CSE \#2 inoperable scenario are obtained through left-right symmetry assumptions and equations. 
3. 6-DOF total aerodynamic force and moment coefficients for the Core, RSRB, and LSRB for nominal BSM operation, but CSE \#4 inoperable.

- The data for the CSE \#1 inoperable scenario are obtained through left-right symmetry assumptions and equations.

\section{A. Coordinate Systems and Booster Separation Parameter Definitions}

The SLS Booster Separation aerodynamic database provides aerodynamic F\&M coefficients for the core stage and the two SRBs in the body axes of the SLS aerodynamic coordinate system. This coordinate system definition is similar to the standard flight mechanics body coordinate system used for airplanes, with the $\mathrm{x}$-axis pointing forward, the $\mathrm{y}$-axis pointing to the right, and the z-axis pointing downwards. Figure 8 shows the SLS aerodynamic coordinate system for the body and missile axes, and also shows the positive sign convention for the aerodynamic F\&M coefficients and the Euler angles.

The position of the SRBs relative to the mated position with the core stage are described by three separation distances $(\Delta X, \Delta Y$, and $\Delta Z)$. The definitions of the separation distances are consistent with the SLS structural coordinate system. The SLS structural coordinate system differs from the SLS aerodynamic coordinate system in that the x-axis points backward and the z-axis points upward. Also, the origin for the SLS structural coordinate system is at a point forward of the nose of the vehicle and was chosen to correspond with the height of the Vehicle Assembly Building at the NASA Kennedy Space Center. The origin of the body axes resides at the moment reference point (MRP) for each of the three bodies. The MRP was chosen to be close to the center of gravity (CG) location for each body during the booster separation event.

The separation distances for the left port SRB (LSRB) and the right starboard SRB (RSRB) are measured in feet from the nose of each booster in the mated position to its instantaneous location during separation. Figure 9 provides a diagram showing the separation distance definitions for the RSRB. Note that the LSRB would have opposite signs from the RSRB for $\Delta Y$ and $\beta$. The figure also shows the origin location and definition of the SLS aerodynamic body axes and the SLS structural coordinate system.

The orientation or attitude of the core stage and the SRBs are described by the angle of attack $(\alpha)$ and the angle of sideslip $(\beta)$ of each body. However, to determine the orientation of the SRBs relative to the core stage orientation, it is easier to use the Euler rotation angles. These relative Euler angles, yaw $(\Delta \psi)$, pitch $(\Delta \theta)$, and roll $(\Delta \phi)$ follow the sign conventions as defined by the aerodynamic body coordinate system (Figure 8-b) and describe the rotation from the core stage body axis to the body axis of each SRB. Note that the LSRB would also have opposite signs from the RSRB for $\Delta \psi$ and $\Delta \phi$. The order of rotation is the standard yaw-pitch-roll or 3-2-1 order meaning a yaw rotation is performed first, followed by a pitch rotation, and finally a roll rotation. 


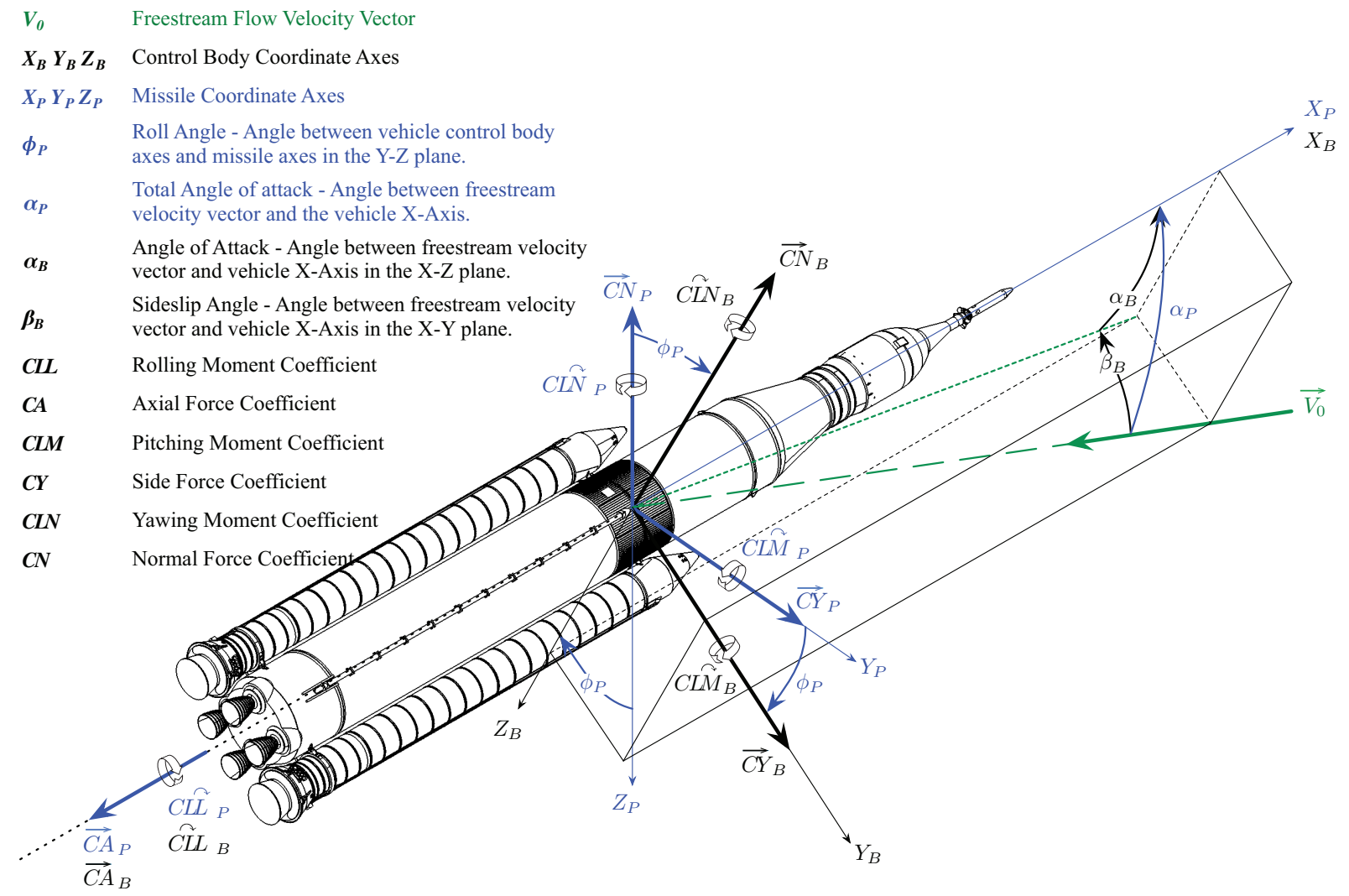

(a) Axis definitions and aerodynamic coefficient sign convention

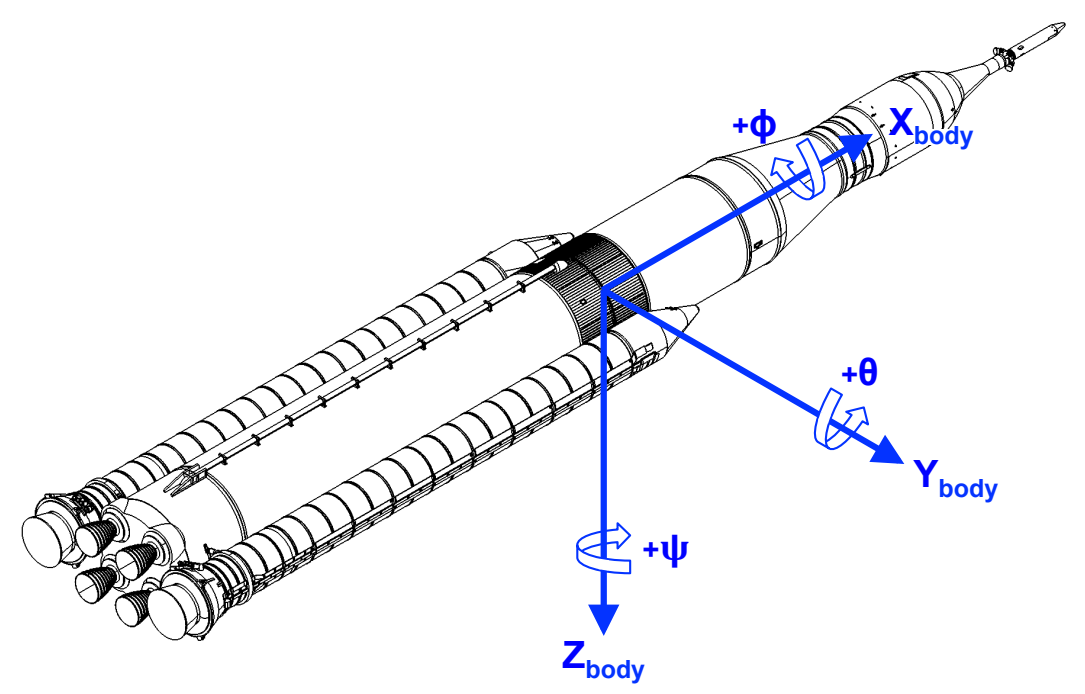

(b) Euler angle sign convention

Figure 8. SLS aerodynamic coordinate system for the body and missile axes. The sign conventions for the (a) aerodynamic coefficients and (b) Euler angles are also provided. 


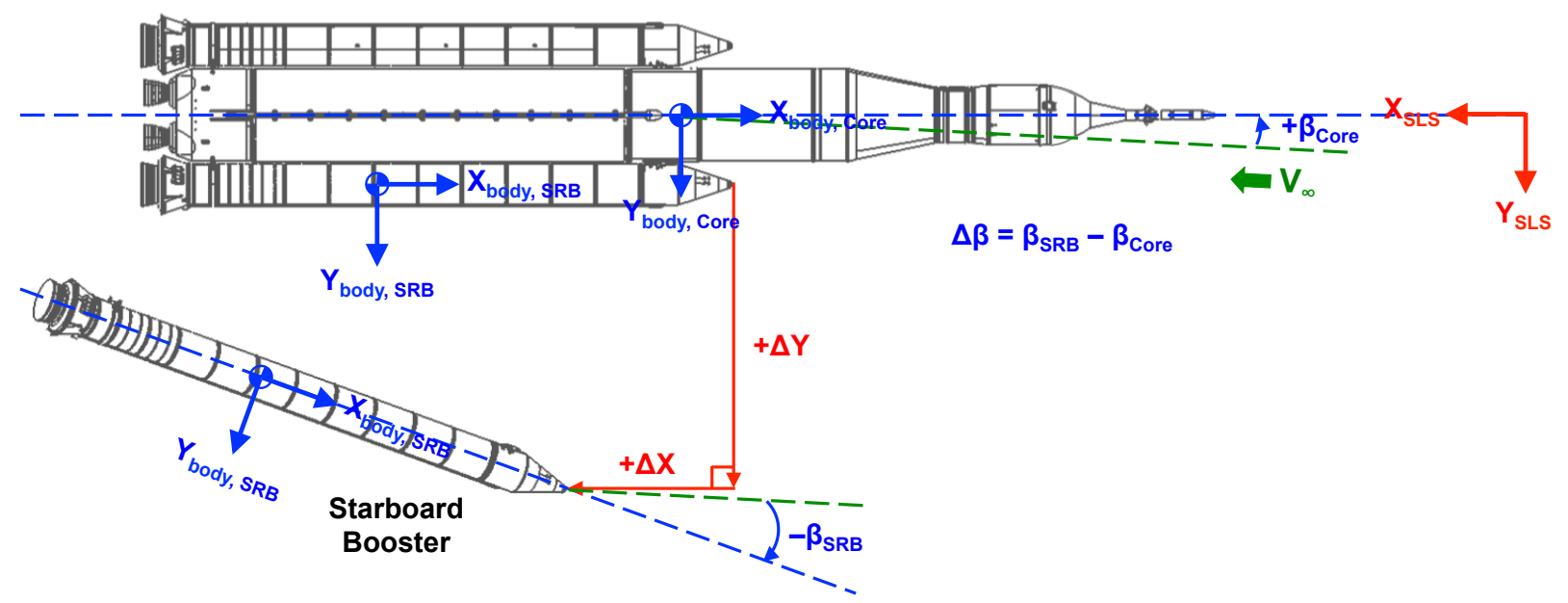

(a) Top view (X-Y plane)

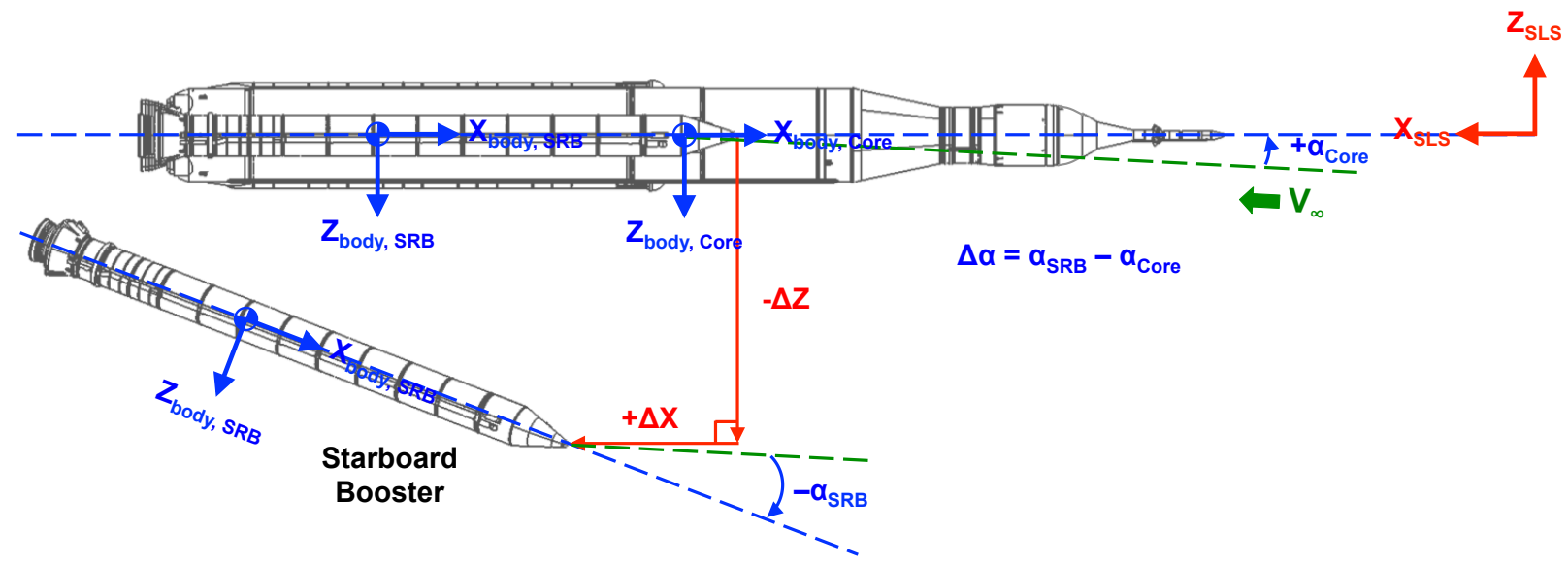

(b) Side view (X-Z plane)

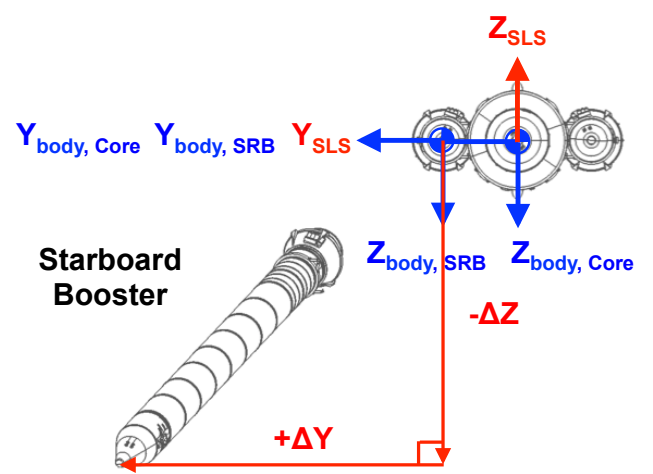

(c) Front view (Y-Z plane)

Figure 9. Definitions for booster separation relative distances shown from the (a) top view, (b) side view, and (c) front view. The distances are measured from the nose of each booster in the mated position to its instantaneous location during separation. Note : The LSRB would have opposite signs from the RSRB for $\Delta Y$ and $\beta$. 


\section{B. Data Space Coverage}

With three separating bodies and multiple engine plumes, the independent parameter space for the booster separation aerodynamic environment is quite expansive. The list of possible independent parameters include:

1. Relative positions for RSRB / LSRB $\left(\Delta X_{R S R B}, \Delta Y_{R S R B}, \Delta Z_{R S R B}, \Delta X_{L S R B}, \Delta Y_{L S R B}, \Delta Z_{L S R B}\right)$

2. Euler rotation angles for $\operatorname{RSRB} / \operatorname{LSRB}\left(\Delta \psi_{R S R B}, \Delta \theta_{R S R B}, \Delta \phi_{R S R B}, \Delta \psi_{L S R B}, \Delta \theta_{L S R B}, \Delta \phi_{L S R B}\right)$

3. Core stage orientation/attitude $\left(\alpha_{\text {core }}, \beta_{\text {core }}\right)$

4. Engine thrust coefficients $\left(C T_{B S M, 1-16}, C T_{R S R B}, C T_{L S R B}, C T_{C S E, 1-4}\right)$

5. Freestream flow conditions $\left(M_{\infty}, q_{\infty}\right)$

However, this results in too many parameters to manage and vary. Therefore, for simplification in the CFD solutions, the RSRB and LSRB are assumed to separate symmetrically so that $\Delta X, \Delta Z$, and $\Delta \theta$ would be the same between the RSRB and LSRB, and $\Delta Y, \Delta \psi$, and $\Delta \phi$ would be equal value but opposite signs between the RSRB and LSRB. Furthermore, the RSRB and LSRB are assumed to be operating at the same thrust level so only one $C T_{S R B}$ parameter is needed. Similarly, all sixteen BSMs are assumed to be operating at the same thrust level and all four CSEs are assumed to be operating at the same thrust level, resulting in just one $C T_{B S M}$ and one $C T_{C S E}$ parameter.

As a result, the booster separation aerodynamic database was chosen to be a function of eight independent parameters: $\Delta X_{R S R B}, \Delta Y_{R S R B}, \Delta Z_{R S R B}, \Delta \psi_{R S R B}, \Delta \theta_{R S R B}, \alpha_{\text {core }}, \beta_{\text {core }}$, and $C T_{B S M}$. Note that only the separation parameters for the RSRB are used since the LSRB separation parameters are symmetric to the RSRB parameters. The BSM thrust coefficient, $C T_{B S M}$, was chosen because the BSM thrust level effected the blockage caused by the BSM plumes and also effected the level of plume impingement on the core stage in the near-proximity region. The SRBs are nearly axisymmetric bodies, therefore the body axis roll angle was assumed to have minimal effect on the total aerodynamic coefficients and thus $\Delta \phi$ was not chosen as an independent parameter. The freestream Mach number, $M_{\infty}$, was set at the nominal booster separation Mach number as specified in the vehicle trajectory. The other parameters $\left(q_{\infty}, C T_{S R B}\right.$, and $\left.C T_{C S E}\right)$ were set at the nominal values as specified in the vehicle trajectory, but were a function of $\Delta X$.

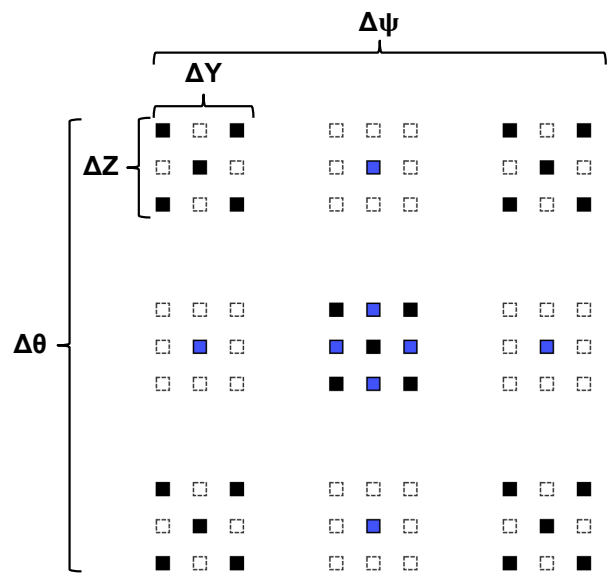

Figure 10. Star-shaped run matrix strategy for Cart3D CFD solutions. ${ }^{8}$
Despite reducing the number of independent parameters to only eight, the nature of the multi-dimensional data space can cause the number of data points needed to cover the entire data space to quickly increase to unmanageable levels. Also, attempting to build a rectangular run matrix for all of the booster separation independent parameters can lead to physically impossible booster positions or intersections of the three bodies, both of which would be a waste of computing resources to simulate. Therefore, a strategy was developed for creating the run matrix for the Cart3D CFD solutions that allowed for sufficient coverage of the data space, but kept the numbers of data points to a reasonable number. ${ }^{8}$ First, the booster axial position variable, $\Delta X$, is used as a special controlling variable for the break points of all the other variables. Then, the run matrix is split into three groups of two: booster positions $\Delta Y$ and $\Delta Z$, booster orientations $\Delta \psi$ and $\Delta \theta$, and core orientation $\alpha_{\text {core }}$ and $\beta_{\text {core }}$. For each pair of variables, five nominal points are chosen in a star pattern which includes the middle value and the four corners as shown by the black points in Figure 10 for the booster position and booster orientation pairs of variables. This pattern is then extended onto the core orientation variables and the blue points are extra points that were chosen only for $\alpha_{\text {core }}=\beta_{\text {core }}=0$.

The break points were selected based on minimum, mean, and maximum values of each variable from Monte Carlo simulations by the SLS GN\&C team using a previous version of the aerodynamic database. The selected break points follow the bounds of the GN\&C trajectory simulations closely and vary as a function of $\Delta X$. For larger values of $\Delta X$ in the far-proximity region, the five-point star pattern can be extended into a thirteen-point pattern where intermediate values between the minimum and maximum values are also 
used. Other points can be chosen as needed, but using the star pattern ensures a good coverage of the data space and allows for easier response surface generation later in the process. The remaining variable $C T_{B S M}$ also has three values (minimum, mean, and maximum) identified, but it is scheduled as a function of $\Delta X$. Figure 11(a) shows an example of the GN\&C simulations bounds for $\Delta Y$ for the nominal flight configuration and how the break points for the CFD solutions were selected.

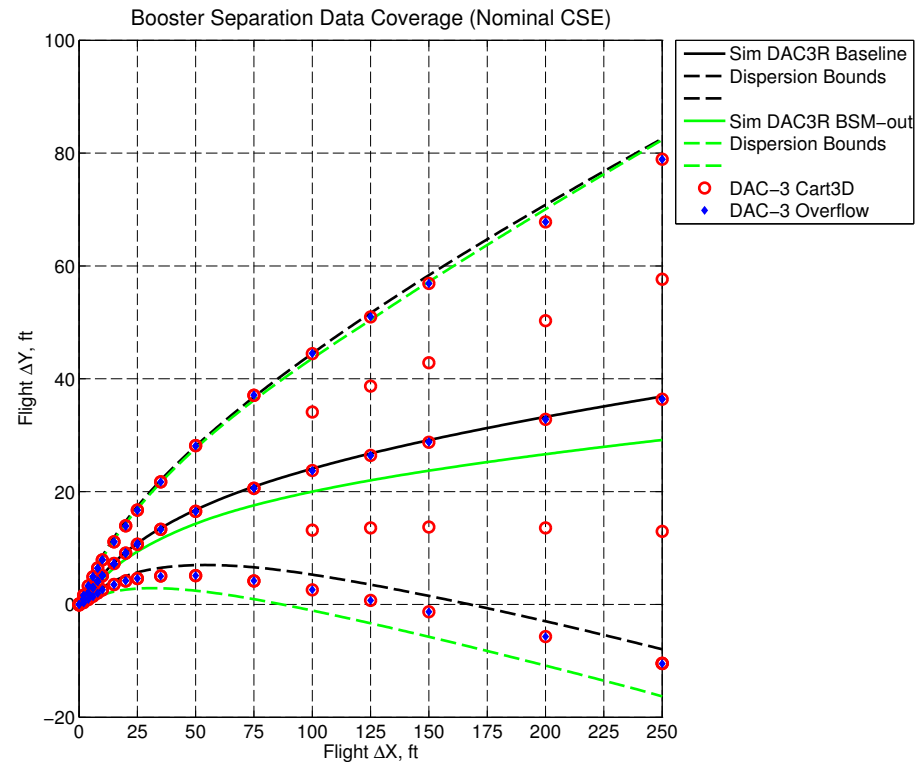

(a) Cart3D and OVERFLOW break points

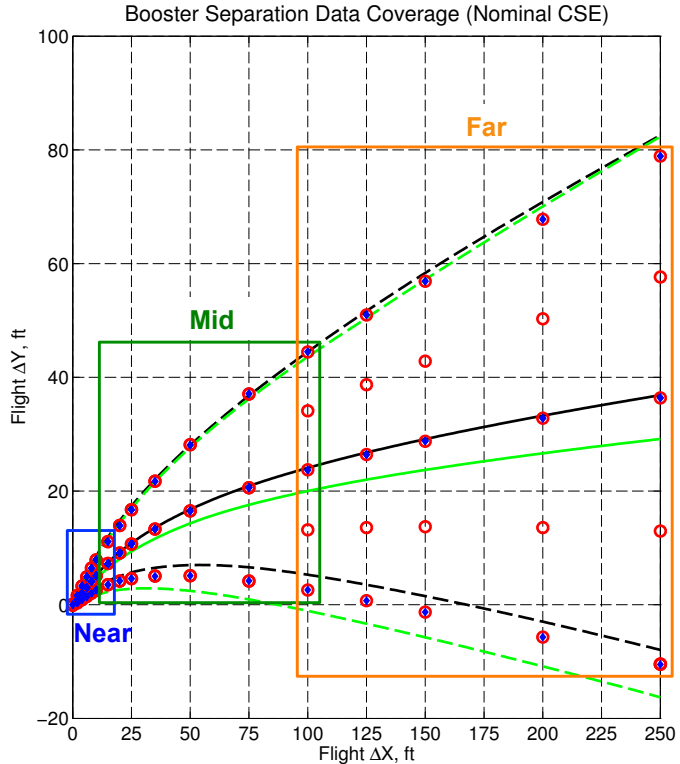

(b) $\Delta X$ regions used for response surface generation

Figure 11. GN\&C trajectory simulation bounds for $\Delta Y$ showing (a) CFD break points for Cart3D and OVERFLOW and (b) regions of $\Delta X$ used for response surface generation.

\section{Database Generation and Response Surface Modeling}

It has already been stated that the initial portion of the booster separation event is most important because of the small clearance distances between the SRBs and the core stage. Additionally, the booster separation data space covers a large portion of longitudinal separation, and the break points for the other independent parameters were chosen to follow the bounds of GN\&C trajectory simulations. Therefore, the development of the aerodynamic database was partitioned into three distinct regions of longitudinal separation: the nearproximity region for $\Delta X$ between 0 and 15 feet, the mid-proximity region for $\Delta X$ between 15 and 100 feet, and the far-proximity region for $\Delta X$ between 100 and 250 feet. Figure 11(b) shows an example for $\Delta Y$ of how the data space is partitioned into regions of $\Delta X$.

The Cart3D CFD solutions were separated into these three $\Delta X$ regions, and multi-dimensional response surfaces were generated for each region. The response surfaces were used to fill in areas where no CFD data existed, either due to the run matrix strategy or due to positions that caused geometry intersections of the three bodies. Response surfaces were generated for each aerodynamic coefficient for each of the three bodies (core and two SRBs) for the nominal flight configuration and the off-nominal one-CSE-out configurations. The multi-dimensional response surfaces were generated using a Matlab ${ }^{\circledR}$-based Kriging response surface generation tool called DACE. ${ }^{9,10}$ The tool provides a constrained non-parametric least-squares fit of a chosen regression and correlation model to the provided input data set. It can handle multiple dimensions at the same time and uses a $0^{\text {th }}, 1^{\text {st }}$, or $2^{\text {nd }}$ order polynomial as the regressor to fit the global trend of the data in a least squares error manner. It also employs several options for a correlation model that controls the influence of one data point to another and forces every input point to be used and fit. For the booster separation response surfaces, the 2nd-order regressor and the exponential correlation model were used.

The response surfaces were then queried at the desired break points of the database to produce the final data tables. The response surfaces were queried at seventeen $\Delta X$ slices between 0 and 250 feet and each $\Delta X$ slice had different break points for the other independent parameters. There were two overlapping $\Delta X$ slices 
between response surfaces, one at 15 feet and one at 100 feet. For these $\Delta X$ slices, both adjacent response surfaces were queried at the overlap $\Delta X$ value and the results were averaged between the two queries. Figure 12 shows an example response surface result for the side force coefficient of the RSRB for $\Delta X=0-75$ feet. The $\Delta X, \Delta Y, \Delta Z$ space is shown and the nominal trajectory is followed for the other independent parameters. It can been seen that the RSRB starts to be affected by the CSE plume impingement in the mid proximity region. Figure 13 shows an example response surface result for the yawing moment coefficient of the RSRB at a $\Delta X$ slice of 2 feet. The star pattern of the Cart3D data points can be seen by the filled black symbols and the colored contours show the response surface results.

The database generation process for the nominal flight configuration is summarized below. A similar process is also used for the off-nominal one-CSE-out configurations.

1. Build response surfaces for each aerodynamic coefficient of the Core, RSRB, and LSRB using the Cart3D data set.

- Separate data into three $\Delta X$ regions

(a) Near-proximity $(\Delta X=0$ to 15 feet $)$

(b) Mid-proximity $(\Delta X=15$ to 100 feet)

(c) Far-proximity $(\Delta X=100$ to 250 feet)

2. Query the response surfaces at seventeen $\Delta X$ slices

- $\Delta X=0,2,4,6,8,10,15,20,25,35,50,75,100,125,150,200,250$ feet

- Each $\Delta X$ slice has different break points for the other independent parameters

- At overlap $\Delta X$ slices of 15 and 100 feet, query both adjacent response surfaces, then average the results

The process results in total aerodynamic coefficient data tables for each of the six aerodynamic coefficients of the three bodies at seventeen $\Delta X$ slices for each of the three engine operation flight configurations. The break points for most of the other independent parameters were different between $\Delta X$ slices. Tables $2-4$ list all of the break points used in the aerodynamic database for the eight independent parameters. Note that only the separation parameters for the RSRB are used since the LSRB separation parameters are symmetric to the RSRB parameters. Also, note that the BSM thrust coefficient $C T_{B S M}$ goes to zero in the far-proximity region. There are a similar set of tables for the one-CSE-out configurations, but they are not listed here.

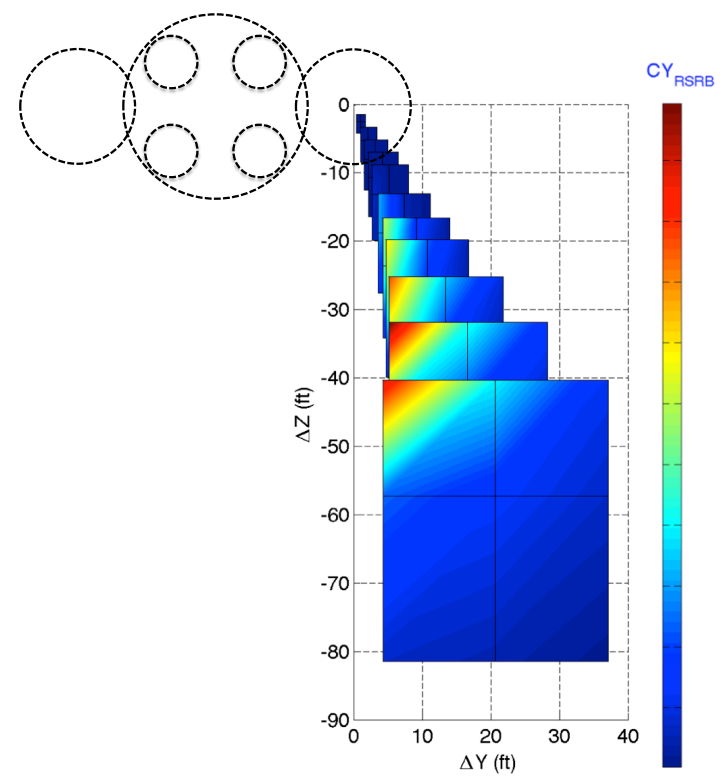

Figure 12. Example response surface result for side force coefficient $(C Y)$ of the RSRB for $\Delta X=0-75$ feet. The RSRB starts to be affected by the CSE plumes in the mid proximity region. 


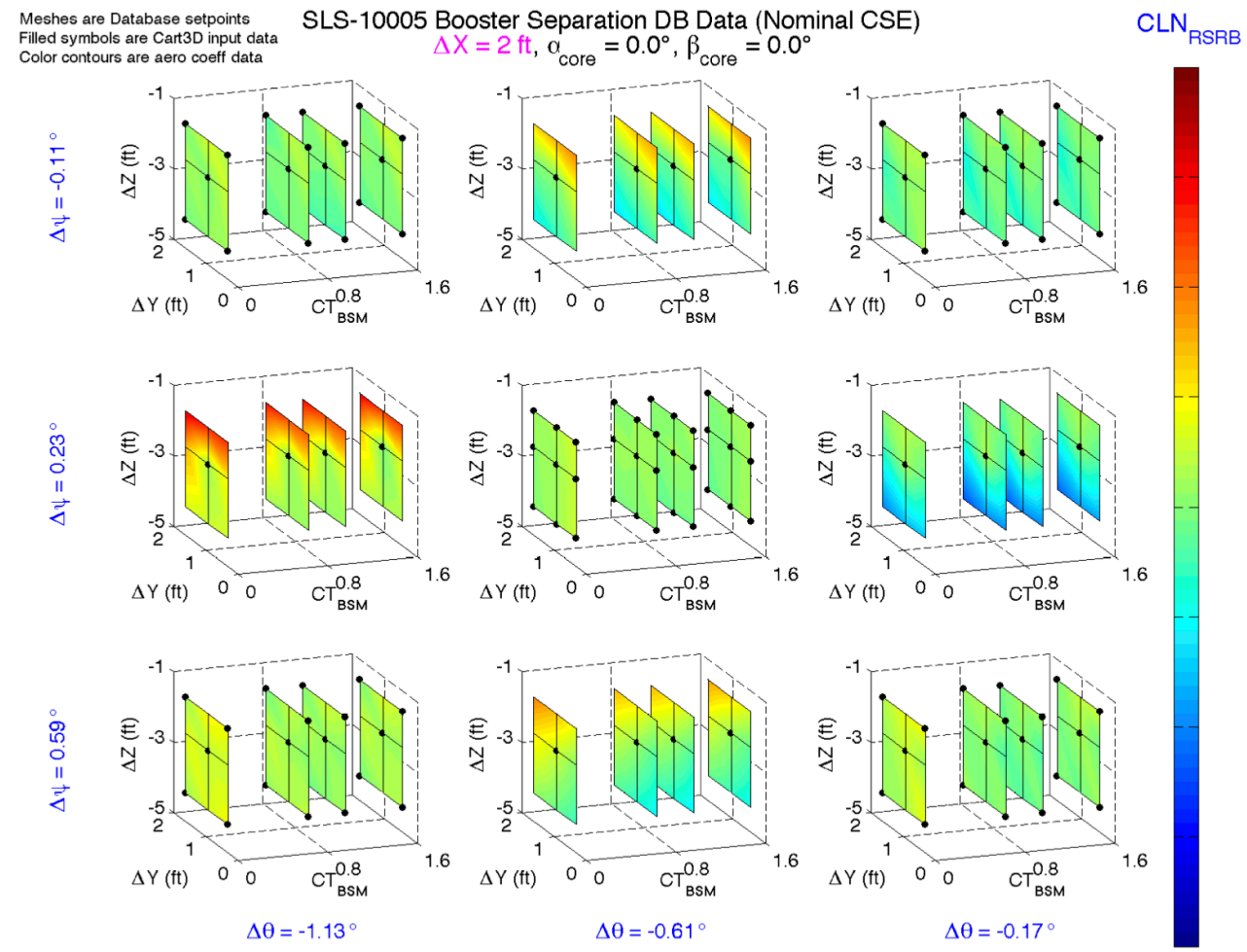

Figure 13. Example response surface results for the yawing moment coefficient $(C L N)$ of the RSRB at $\triangle X=$ 2 feet. The meshes are the database break points, the filled black symbols are the Cart3D input data, and the colored contours are the response surface results.

Table 2. $\Delta Y$ and $\Delta Z$ database break points for nominal flight configuration.

\begin{tabular}{|c|c|c|}
\hline$\Delta X_{R S R B}, \mathrm{ft}$ & $\Delta Y_{R S R B}, \mathrm{ft}$ & $\Delta Z_{R S R B}, \mathrm{ft}$ \\
\hline 0 & $-0.10,0,0.10$ & $-0.10,0,0.10$ \\
\hline 2 & $0.33,0.93,1.64$ & $-4.20,-2.50,-1.46$ \\
\hline 4 & $0.88,1.98,3.27$ & $-8.46,-5.26,-3.29$ \\
\hline 6 & $1.45,3.03,4.89$ & $-12.55,-7.99,-5.14$ \\
\hline 8 & $2.03,4.07,6.44$ & $-16.39,-10.65,-6.99$ \\
\hline 10 & $2.57,5.06,7.89$ & $-19.90,-13.19,-8.82$ \\
\hline 15 & $3.52,7.25,11.09$ & $-27.56,-18.82,-13.03$ \\
\hline 20 & $4.16,9.09,13.93$ & $-34.12,-23.64,-16.64$ \\
\hline 25 & $4.60,10.66,16.71$ & $-39.94,-27.90,-19.81$ \\
\hline 35 & $5.05,13.31,21.72$ & $-50.13,-35.29,-25.23$ \\
\hline 50 & $5.10,16.50,28.15$ & $-63.15,-44.57,-31.88$ \\
\hline 75 & $4.16,20.58,37.06$ & $-81.43,-57.24,-40.39$ \\
\hline 100 & $2.58,13.16,23.74,34.10,44.46$ & $-97.27,-82.60,-67.93,-57.12,-46.32$ \\
\hline 125 & $0.71,13.56,26.41,38.69,50.97$ & $-111.71,-94.57,-77.43,-64.31,-51.20$ \\
\hline 150 & $-1.30,13.72,28.75,42.83,56.90$ & $-125.19,-105.66,-86.12,-70.73,-55.35$ \\
\hline 200 & $-5.69,13.56,32.81,50.30,67.80$ & $-150.08,-126.01,-101.95,-81.95,-61.95$ \\
\hline 250 & $-10.47,12.95,36.37,57.64,78.92$ & $-173.21,-144.84,-116.46,-91.72,-66.99$ \\
\hline
\end{tabular}


Table 3. $\Delta \psi$ and $\Delta \theta$ database break points for nominal flight configuration.

\begin{tabular}{|c|c|c|}
\hline$\Delta X_{R S R B}, \mathrm{ft}$ & $\Delta \psi_{R S R B}, \mathrm{deg}$ & $\Delta \theta_{R S R B}, \mathrm{deg}$ \\
\hline 0 & $-0.10,0,0.10$ & $-0.10,0,0.10$ \\
\hline 2 & $-0.11,0.23,0.59$ & $-1.13,-0.61,-0.17$ \\
\hline 4 & $-0.10,0.50,1.11$ & $-2.18,-1.28,-0.50$ \\
\hline 6 & $-0.08,0.76,1.01,1.61$ & $-3.20,-1.94,-0.82$ \\
\hline 8 & $-0.06,1.01,1.35,2.09$ & $-4.18,-2.58,-1.14$ \\
\hline 10 & $-0.05,1.26,1.75,2.54$ & $-5.09,-3.18,-1.46$ \\
\hline 15 & $-0.09,1.79,2.50,3.67$ & $-7.09,-4.52,-2.20$ \\
\hline 20 & $-0.20,2.19,3.00,4.67$ & $-8.82,-5.66,-2.81$ \\
\hline 25 & $-0.38,2.50,3.25,5.56$ & $-10.36,-6.67,-3.33$ \\
\hline 35 & $-0.88,2.94,3.75,7.16$ & $-13.08,-8.41,-4.15$ \\
\hline 50 & $-1.90,3.34,4.25,9.15$ & $-16.55,-10.52,-5.03$ \\
\hline 75 & $-4.08,3.52,5.00,10.50,11.84$ & $-21.36,-13.17,-5.93$ \\
\hline 100 & $-6.63,-1.66,3.32,8.62,13.92$ & $-25.45,-20.32,-15.18,-10.78,-6.37$ \\
\hline 125 & $-9.32,-3.18,2.96,9.31,15.66$ & $-28.95,-22.88,-16.80,-11.67,-6.53$ \\
\hline 150 & $-11.94,-4.71,2.52,9.85,17.18$ & $-32.09,-25.12,-18.16,-12.20,-6.23$ \\
\hline 200 & $-16.96,-7.70,1.56,10.64,19.72$ & $-37.86,-29.13,-20.41,-12.83,-5.25$ \\
\hline 250 & $-21.76,-10.56,0.64,11.33,22.01$ & $-43.31,-32.86,-22.40,-13.21,-4.01$ \\
\hline
\end{tabular}

Table 4. $\alpha_{\text {core }}, \beta_{\text {core }}$, and $C T_{B S M}$ database break points for nominal flight configuration.

\begin{tabular}{|c|c|c|c|}
\hline$\Delta X_{R S R B}, \mathrm{ft}$ & $\alpha_{\text {core }}, \mathrm{deg}$ & $\beta_{\text {core }}, \mathrm{deg}$ & $C T_{B S M}$ \\
\hline 0 & $-2.50,0,2.50$ & $-3.50,0,3.50$ & $0,0.71,1.06,1.55$ \\
\hline 2 & $-2.50,0,2.50$ & $-3.50,0,3.50$ & $0,0.72,1.05,1.56$ \\
\hline 4 & $-2.50,0,2.50$ & $-3.50,0,3.50$ & $0,0.73,1.04,1.56$ \\
\hline 6 & $-2.50,0,2.50$ & $-3.50,0,3.50$ & $0,0.73,1.03,1.35$ \\
\hline 8 & $-2.50,0,2.50$ & $-3.50,0,3.50$ & $0,0.33,0.90,1.17$ \\
\hline 10 & $-2.50,0,2.50$ & $-3.50,0,3.50$ & $0,0.17,0.52,1.14$ \\
\hline 15 & $-2.50,0,2.50$ & $-3.50,0,3.50$ & $0,0.16,0.44$ \\
\hline 20 & $-2.50,0,2.50$ & $-3.50,0,3.50$ & $0,0.09,0.18$ \\
\hline 25 & $-2.50,0,2.50$ & $-3.50,0,3.50$ & $0,0.05,0.11$ \\
\hline 35 & $-2.50,0,2.50$ & $-3.50,0,3.50$ & $0,0.06$ \\
\hline 50 & $-2.50,0,2.50$ & $-3.50,0,3.50$ & $0,0.03$ \\
\hline 75 & $-2.50,0,2.50$ & $-3.50,0,3.50$ & $0,0.02$ \\
\hline 100 & $-2.50,0,2.50$ & $-3.50,0,3.50$ & 0 \\
\hline 125 & $-2.50,0,2.50$ & $-3.50,0,3.50$ & 0 \\
\hline 150 & $-2.50,0,2.50$ & $-3.50,0,3.50$ & 0 \\
\hline 200 & $-2.50,0,2.50$ & $-3.50,0,3.50$ & 0 \\
\hline 250 & $-2.50,0,2.50$ & $-3.50,0,3.50$ & 0 \\
\hline & & & \\
\hline
\end{tabular}




\section{Database Uncertainty Quantification}

In addition to the total aerodynamic coefficients provided in the database, the uncertainties associated with the aerodynamic coefficients were also developed. There are uncertainty tables for the three engine operation scenarios (nominal, CSE3-out, CSE4-out) and also another set of three uncertainty tables to cover the one-BSM-out scenarios. The extra one-BSM-out uncertainty tables were included because there was not a separate database developed for the one-BSM-out scenario. Each uncertainty table is a function of $\Delta X$ and is separated into four ranges $(0-10$ feet, $15-25$ feet, $35-75$ feet, and $100-250$ feet $)$ to focus on the near, mid, and far proximity distances, and especially to keep uncertainties smaller in the critical near-proximity area. The four ranges do not overlap in order to prevent instantaneous step changes in the uncertainty values. Within each $\Delta X$ range, the uncertainty values are constant and linear interpolation is used to obtain values between ranges. The uncertainties are also developed at the MRP of each body.

\section{A. Overall Strategy}

The overall strategy for the booster separation database uncertainty quantification (UQ) process is an attempt to capture three categories of errors between the CFD-based database and the actual flight environment: model-form errors, numerical errors, and database modeling errors as shown in Figure 14. Model-form errors pertain to any errors or mismodeling of the physics of the booster separation aerodynamic environment in the CFD solutions. Numerical errors pertain to the mathematics of the CFD flow solver and can include iterative convergence error, statistical sampling error, discretization error, and round-off error. Finally, database modeling errors pertain to errors associated with database assumptions or modeling decisions.

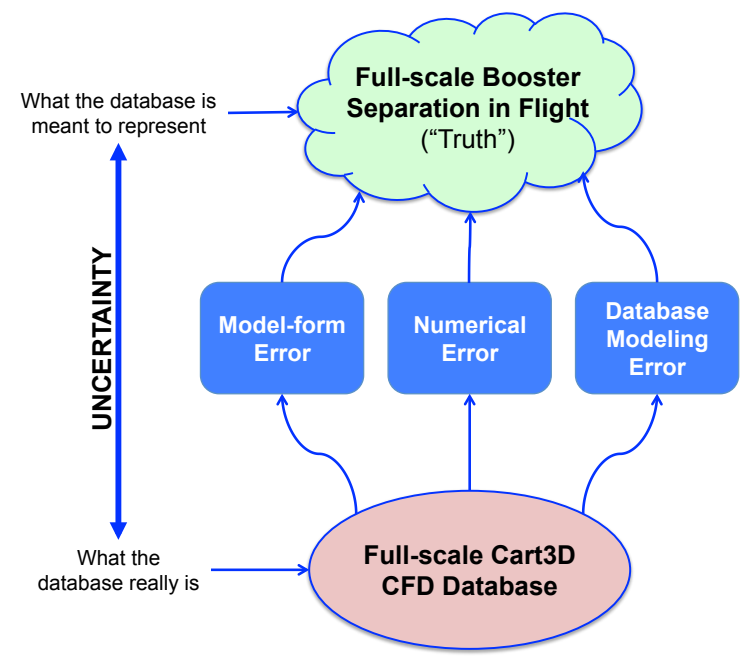

Figure 14. Uncertainty modeling strategy for SLS Booster Separation aerodynamic database.

For the booster separation database, the uncertainty model attempts to cover errors in the source Cart3D data and errors in the database modeling process. It also includes additional bump-up factors for unmodeled or unknown effects. Within this model, each error category described above is also addressed. The uncertainty model is summarized below:

- Errors associated with source Cart3D CFD data

- Model-form errors

* Code-to-WT comparison including baseline WT uncertainty (Cart3D vs. UPWT)

* Code-to-Code comparison between inviscid and viscous flow solvers (Cart3D vs. OVERFLOW)

- Numerical errors

* Statistical Sampling (Averaging over last X iterations of solution)

- Database Modeling Errors 
- Interpolation errors due to discretization of data set

- Symmetry assumptions ( $\beta$ symmetry, RSRB vs LSRB, CSE-out symmetry)

- One-BSM-out induced aerodynamic effect (One-BSM-out vs. Nominal)

- Additional factors for unmodeled or unknown effects

- Sensitivities to other unmodeled independent parameters $\left(M_{\infty}, q_{\infty}, C T_{S R B}, C T_{C S E}, \Delta \phi\right)$

- Unknown effects

\section{B. Development}

The uncertainty buildup equations shown in Equation 1 for the nominal and one-CSE-out flight configurations and Equation 2 for the one-BSM-out configurations contain root-sum-square (RSS) of multiple error terms $\left(\sigma_{C x}\right)$ related to the Cart3D uncertainty and database development uncertainty. The $C x$ nomenclature equates to each of the six aerodynamic coefficients. The CFD-related terms are the Code-to-WT (Cart3D vs. UPWT), the Code-to-Code (Cart3D vs. OVERFLOW), and the Sampling (Cart3D iteration sampling) terms. The database-related terms are the Interpolation, Symmetry, and BSM-out terms.

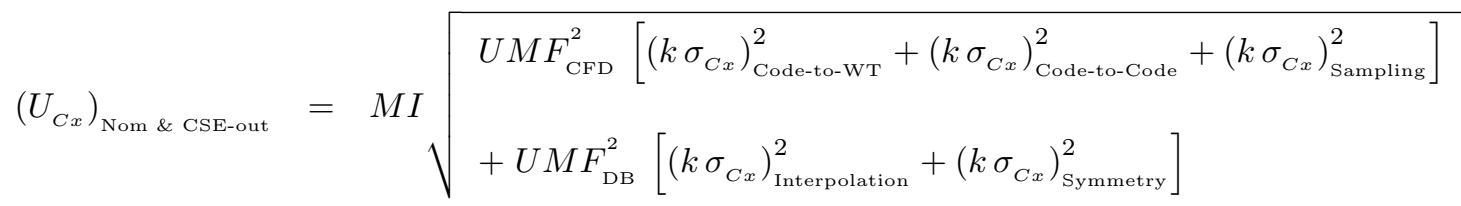

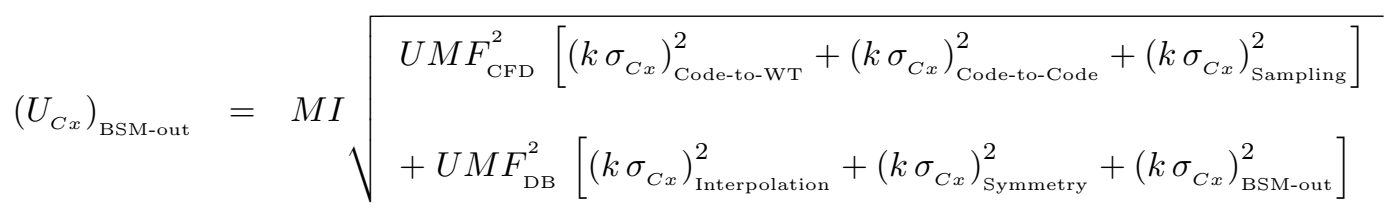

$$
\begin{aligned}
& \text { where } C x=\mathrm{CA}, \mathrm{CN}, \mathrm{CY}, \mathrm{CLL}, \mathrm{CLM} \text {, and CLN }
\end{aligned}
$$

Each term has an associated coverage factor $(k)$ that is chosen based on range analysis results to cover $\geq 99.7 \%$ of the analysis residuals. There is also an Uncertainty Model Factor $(U M F \geq 1)$ for the CFDrelated terms and the database-related terms to account for unmodeled or unknown errors. The $U M F$ factors are just multiplication bump-up factors to the uncertainty. For the CFD-related uncertainty terms, a $30 \%$ bump-up factor $\left(U M F_{C F D}=1.3\right)$ was chosen because although an attempt was made to compare the Cart3D data to WT data and to OVERFLOW data, there may be additional model-form errors that are unaccounted. For the database-related uncertainty terms, a $10 \%$ bump-up factor $\left(U M F_{D B}=1.1\right)$ was chosen because only a limited set of interpolation check points were available and could not cover the entire data space adequately. Finally, there is a total margin index $(M I \geq 1)$ on the outside of the equation that allows the program technical lead to provide additional margin to the uncertainty levels if necessary. Figure 15 shows a graphical representation of the uncertainty buildup equation and how it relates to the overall strategy.

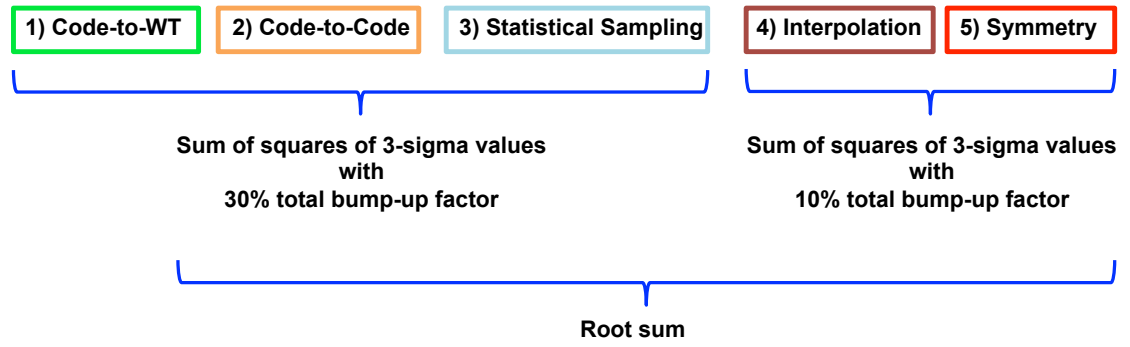

Figure 15. Uncertainty modeling terms and buildup. 
A brief description of the development of each of the uncertainty terms is provided in the following sections. The uncertainty in each term (except for the Cart3D iteration sampling) is determined using data analysis techniques commonly used in Statistical Process Control (SPC).${ }^{11-16}$ The Analysis of Normalized Ranges with mean ranges $(\bar{R})$ is used because of its simplicity and also because of its ability to handle varying numbers of data points within a group. For each of the uncertainty terms of interest, data sets are gathered for comparison and the range analysis provides a measure of how well the data sets compare to each other. For each comparison data set, the analysis process is described below.

1. Interpolate each data set to nominal independent variable (e.g. $\Delta X$ ) values $x_{i}$, if necessary;

2. For the dependent variable of interest (e.g. $C Y$ ), calculate the mean of the $y_{i}$ values at each $x_{i}$;

3. Calculate the residuals from the mean for each $y_{i}$ value;

4. Calculate the range of the $y_{i}$ residuals $(R=\max -\min )$ at each $x_{i}$;

5 . Normalize each individual range by the quantity $d_{2}$ to produce an individual dispersion quantity $\left(R / d_{2}\right)$

The quantity $d_{2}$ relates the mean range for a normal distribution to its standard deviation and is a function of the sample size as listed in Table 5. The individual normalized ranges are gathered and can be organized and grouped by independent variables such as $\Delta X$. The mean of the normalized ranges produces an estimated standard deviation (1- $\sigma)$ as shown in Equation 3. An overall mean range for the complete data set is calculated, but mean ranges can also be calculated as a function of each independent variable to determine if any trends exist and help determine the source of variability in the data set. In fact, for many of the booster separation uncertainty terms, the trend with $\Delta X$ was used.

$$
\sigma=\frac{\bar{R}}{d_{2}}
$$

The standard deviation estimate using the mean of the normalized ranges is a statistical measure of the data set. In order to determine the coverage factor $(k)$ that will ensure that the standard deviation estimate covers at least $99.7 \%$ of the analysis residuals, histograms of the residuals are plotted for both the entire data set and for the residuals grouped by independent parameter values. The standard deviation estimates are plotted on top of these histograms and the coverage factor can then be determined.

Table 5. Example values of range analysis quantity $d_{2}$.

\begin{tabular}{|c|c|}
\hline Number of Samples & Value of $d_{2}$ \\
\hline 2 & 1.128 \\
\hline 3 & 1.693 \\
\hline 4 & 2.059 \\
\hline 5 & 2.326 \\
\hline
\end{tabular}

\section{UQ Term for Cart3D to UPWT Comparison}

This term looked at the comparison between the UPWT data and the Cart3D simulations of the WT geometry and BSM plume simulation using high pressure air. In the UPWT test when the BSMs were active, there were two main core nose bow shock locations: one location near the abort motor nozzles on the launch abort system and one location on the MPCV capsule. ${ }^{7}$ The location was dependent on the position and attitude of the SRBs relative to the core stage and it was determined that for the bounds of the GN\&C trajectory simulations, the shock location on the MPCV capsule was the more likely location for the actual flight environment. Also, blockage from the separation rig support system caused an increase in the core base pressures, which likely corrupted the data. Therefore, for this comparison, only UPWT data points where the bow shock location was on the MPCV capsule and where the core base pressures were less than the freestream static pressure were used. This led to a total of 2,798 data points to be used for comparison. Overall, the comparison between the Cart3D data and the UPWT data were very good, especially for the SRBs. Figure 16 shows an example comparison between the two data sets for the core and the RSRB. The uncertainty estimates for each data source are also shown for reference. 


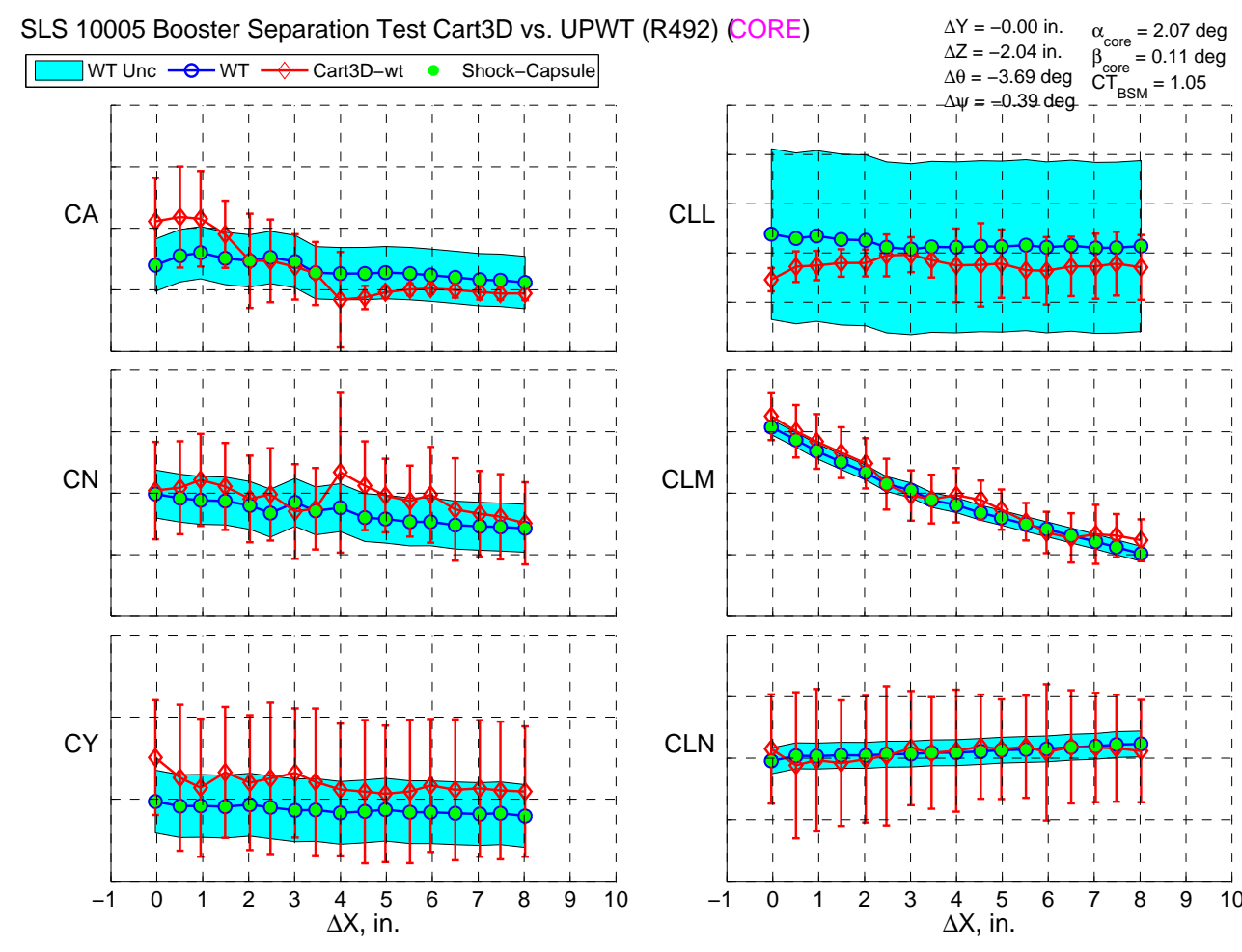

(a) Core
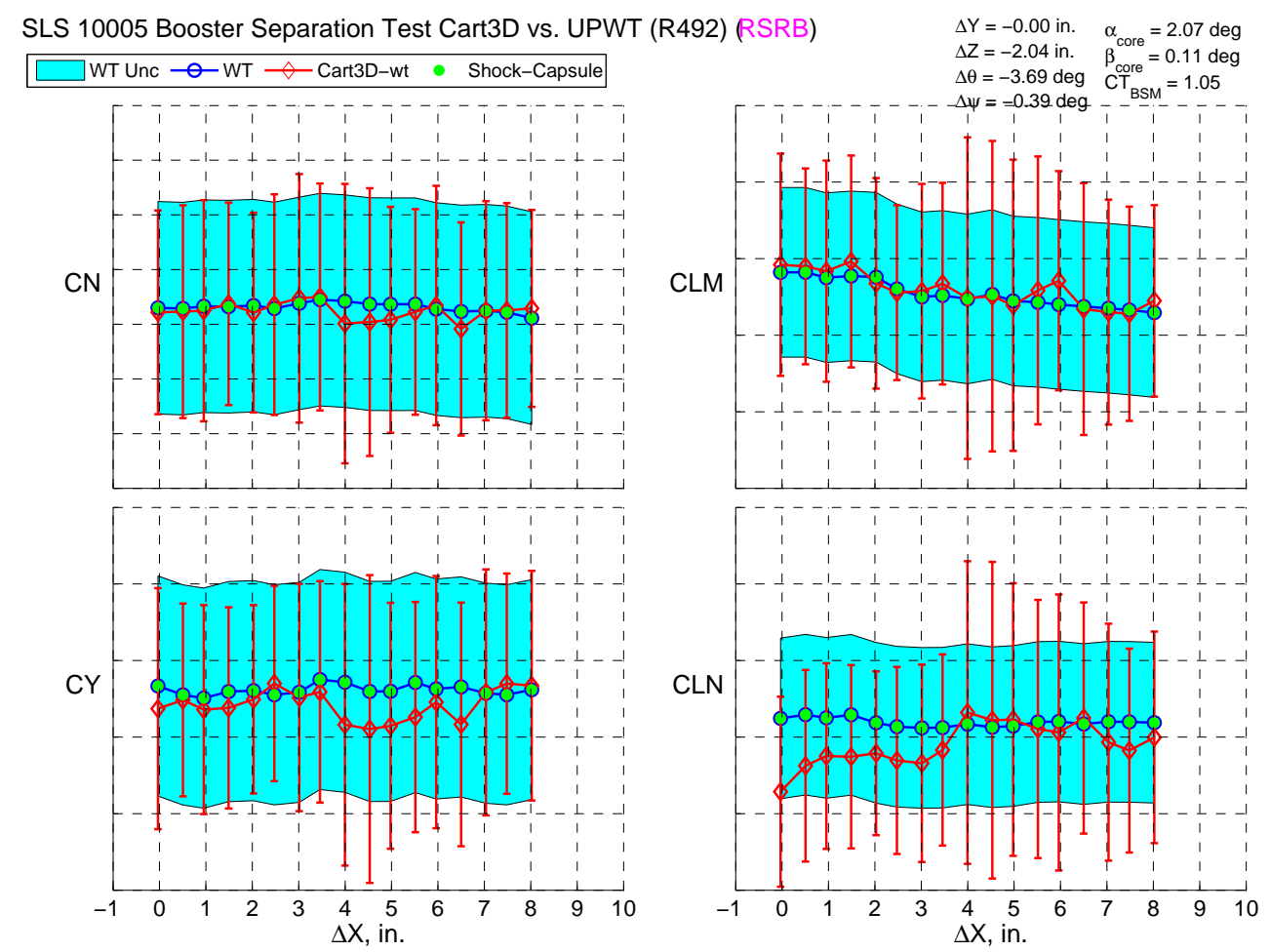

(b) RSRB

Figure 16. Example comparison between UPWT and Cart3D data for the aerodynamic coefficients of the (a) Core and (b) RSRB. Note : The UPWT test did not measure axial force or rolling moment on the SRBs. 


\section{UQ Term for Cart3D to OVERFLOW Comparison}

This term looked at the comparison between the Cart3D and OVERFLOW simulations of the flight geometry including simulation of all engine plumes between the BSMs, SRBs, and CSEs. There were a limited amount of OVERFLOW solutions acquired due to the amount of time and resources needed for each booster separation solution. This amounted to a total of 232 comparison data points for the nominal flight configuration and 82 comparison data points each for the CSE3-out and CSE4-out configurations. For the core stage, the comparisons were better in the mid and far proximity regions than they were in the near proximity region, most likely due to differences in predicted BSM plume effects. For the SRBs, the comparisons were good in the near and far proximity regions, but suffered in the mid proximity region, most likely due to differences in predictions of CSE plume impingement on the SRBs. Figure 17 shows an example comparison between the two data sets for the core and the RSRB. The uncertainty estimates for each data source are also shown for reference.

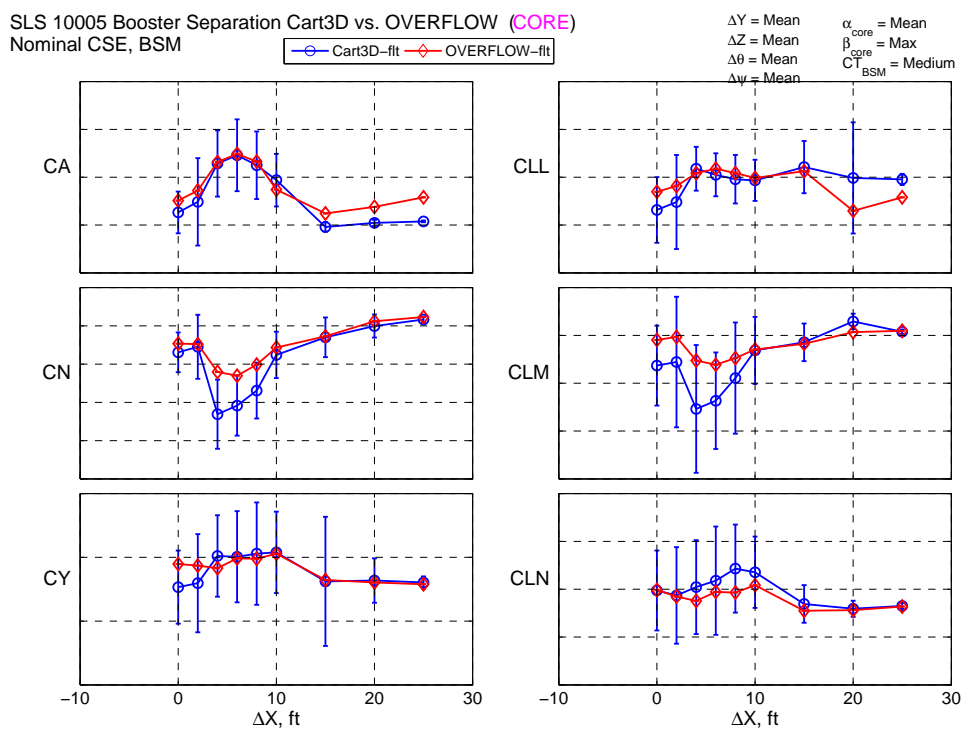

(a) Core

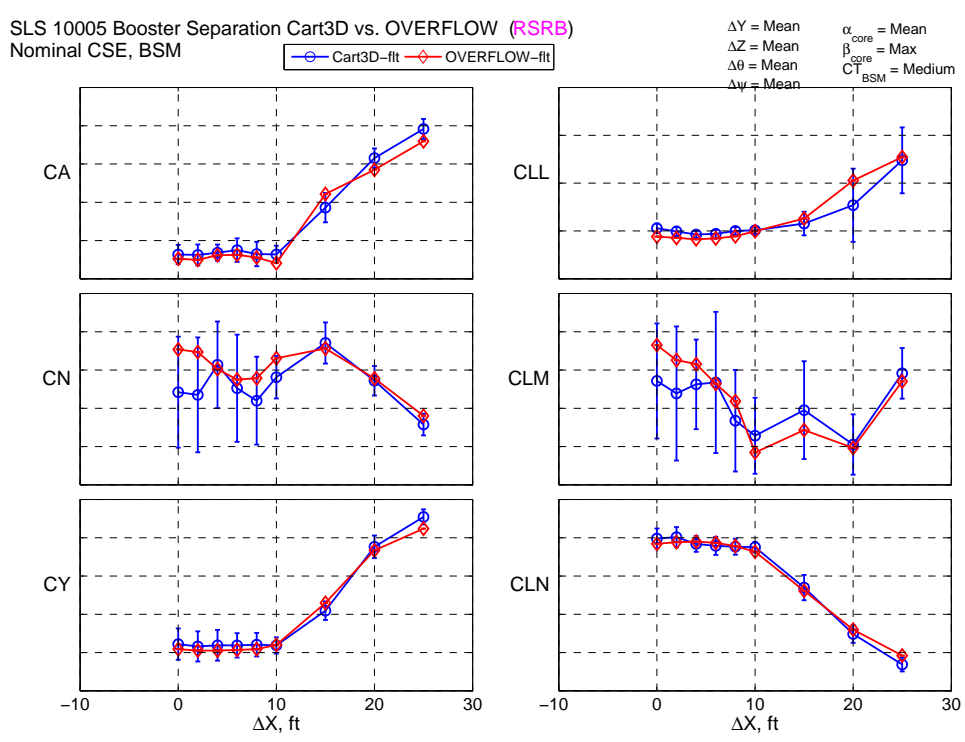

(b) RSRB

Figure 17. Example comparison between Cart3D and OVERFLOW data for the aerodynamic coefficients of the (a) Core and (b) RSRB. 


\section{UQ Term for Cart3D Iteration Sampling}

This term provided a conservative estimate of the statistical sampling uncertainty in the Cart3D solutions by calculating the standard deviation of all the samples used in the solution average of the last $\mathrm{X}$ iterations. The standard deviation for all of the Cart3D solutions were averaged and then multiplied by 3 for coverage and used in the uncertainty buildup equation. Figure 18 shows statistics for an example Cart3D booster separation solution and in particular, the standard deviation of the iteration samples used in the solution average.

\begin{tabular}{|c|c|c|c|c|c|c|}
\hline \multicolumn{3}{|l|}{ Conditions } & \multicolumn{4}{|c|}{ Force \& moment summary (Iteration $6000, n S$ tats $=2000$ ) } \\
\hline Variable & Abbr. & Value & Coefficient & CORE_No_Base & LSRB_No_Base & RSRB_No_Base \\
\hline$d x$ & $d x$ & 4.0 & $C A$ mean, $\mu\left(C_{A}\right)$ & & & \\
\hline dy & dy & 0.88 & $C A$ standard deviation, $\sigma\left(C_{A}\right)$ & & & \\
\hline$d z$ & $d z$ & -8.46 & $C Y$ mean, $\mu\left(C_{Y}\right)$ & & & \\
\hline dpsi & dps & -0.1 & $C Y$ standard deviation, $\sigma\left(C_{Y}\right)$ & & & \\
\hline dtheta & dth & -0.5 & $C Y$ iterative uncertainty, $\varepsilon\left(C_{Y}\right)$ & & & \\
\hline dphi & dphi & 0.0 & $C N$ mean, $\mu\left(C_{N}\right)$ & & & \\
\hline alpha & a & -2.5 & $C N$ standard deviation, $\sigma\left(C_{N}\right)$ & & & \\
\hline beta & $\mathrm{b}$ & -3.5 & $C N$ iterative uncertainty, $\varepsilon\left(C_{N}\right)$ & & & \\
\hline mach & $\mathrm{m}$ & 4.25 & $C L L$ mean, $\mu\left(C_{\ell}\right)$ & & & \\
\hline q & q & 36.7 & $C L L$ standard deviation, $\sigma\left(C_{\ell}\right)$ & & & \\
\hline CT_CSE & CT_CSE & 21.18 & $C L M$ mean, $\mu\left(C_{m}\right)$ & & & \\
\hline CT_SRB & CT_SRB & 1.1 & CLM standard deviation, $\sigma\left(C_{m}\right)$ & & & \\
\hline $\begin{array}{l}\text { CT_BSM } \\
\text { config }\end{array}$ & CT & $\begin{array}{c}1.04 \\
\text { bsmon }\end{array}$ & $\begin{array}{l}C L N \text { mean, } \mu\left(C_{n}\right) \\
C L N \text { standard deviation, } \sigma\left(C_{n}\right)\end{array}$ & & & \\
\hline
\end{tabular}
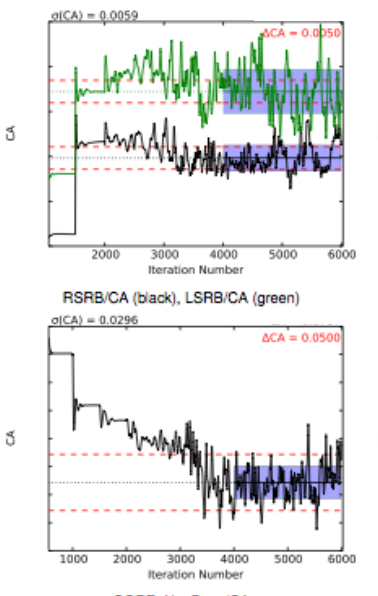

CORE_No_Base/CA



RSRB_No_Base/CY

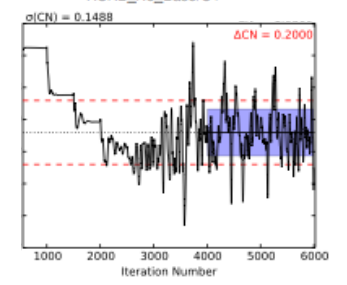

CORE_ No_Base/CN

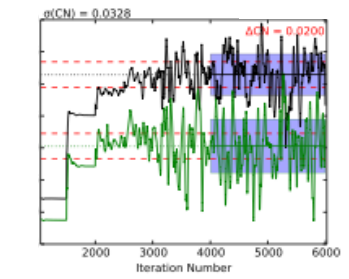

SAB/CN

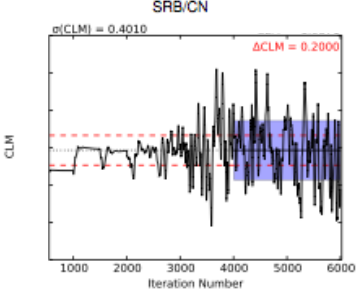

CORE_No_Base/CLM

Figure 18. Example Cart3D solution statistics.

\section{UQ Term for Database Interpolation}

This term is intended to quantify the error in the response surface fit to the input Cart3D data and the error in the discretization of the data set required for GN\&C use. A small set of validation CFD solutions at points in between the database break points were used to test the response surface fit and database interpolation. A total of 54 points were used for the nominal flight configuration and 50 total points each for the CSE3-out and CSE4-out configurations. Note that these points were not used in the generation of the response surfaces for the database.

\section{UQ Term for Symmetry Assumption}

This term looked at the error associated with the left-right vehicle symmetry assumption used in the database. For the nominal flight configuration, this assumption allowed data to be mirrored across $\beta_{\text {core }}$ and allowed RSRB data to be mirrored for the LSRB. A total of 8,866 points from the Cart3D data set were used to test the assumption and how well the data followed the symmetry rules as shown in Equation 4 for the core stage and Equation 5 for the SRBs. 


$$
\begin{aligned}
(C Y)_{\text {Core }, \beta=0} & =0 \\
(C L L)_{\text {Core }, \beta=0} & =0 \\
(C L N)_{\text {Core }, \beta=0} & =0 \\
(C A)_{\text {Core },+\beta} & =(C A)_{\text {Core },-\beta} \\
(C N)_{\text {Core },+\beta} & =(C N)_{\text {Core },-\beta} \\
(C L M)_{\text {Core },+\beta} & =(C L M)_{\text {Core },-\beta} \\
(C Y)_{\text {Core },+\beta} & =-(C Y)_{\text {Core },-\beta} \\
(C L L)_{\text {Core },+\beta} & =-(C L L)_{C o r e,-\beta} \\
(C L N)_{\text {Core },+\beta} & =-(C L N)_{C o r e,-\beta} \\
(C A)_{R S R B, \beta=0} & =(C A)_{L S R B, \beta=0} \\
(C N)_{R S R B, \beta=0} & =(C N)_{L S R B, \beta=0} \\
(C L M)_{R S R B, \beta=0} & =(C L M)_{L S R B, \beta=0} \\
(C Y)_{R S R B, \beta=0} & =-(C Y)_{L S R B, \beta=0} \\
(C L L)_{R S R B, \beta=0} & =-(C L L)_{L S R B, \beta=0} \\
(C L N)_{R S R B, \beta=0} & =-(C L N)_{L S R B, \beta=0} \\
(C A)_{R S R B,+\beta} & =(C A)_{L S R B,-\beta} \\
(C N)_{R S R B,+\beta} & =(C N)_{L S R B,-\beta} \\
(C L M)_{R S R B,+\beta} & =(C L M)_{L S R B,-\beta} \\
(C Y)_{R S R B,+\beta} & =-(C Y)_{L S R B,-\beta} \\
(C L L)_{R S R B,+\beta} & =-(C L L)_{L S R B,-\beta} \\
(C L N)_{R S R B,+\beta} & =-(C L N)_{L S R B,-\beta}
\end{aligned}
$$

For the one-CSE-out configurations, the symmetry assumption allowed data for the CSE3-out configuration to be mirrored for the CSE2-out configuration, and similarly for CSE4-out and CSE1-out configurations. A total of only 8 points each were run for the CSE1-out and CSE2-out configurations to test the symmetry assumption. The symmetry rules are similar to those used for the nominal flight configuration except that the RSRB from CSE3-out is symmetric to the LSRB from CSE2-out and vice versa. The same is true for CSE4-out and CSE1-out.

\section{UQ Term for One-BSM-out Scenario}

This term is included because there is not a separate force \& moment database for the one-BSM-out configuration. It is intended to quantify the effect of a one-BSM-out configuration in the near proximity region and it is intended to evaluate the expanded GN\&C trajectory simulation bounds for the one-BSM-out configuration in the mid and far proximity regions. A total of 75 data points were acquired and these data points were compared to the data points from the nominal flight configuration.

\section{Results}

In GN\&C simulations of previous iterations of the booster separation aerodynamic database, it was determined that the likelihood of re-contact between the boosters and core stage was very sensitive to the aerodynamic uncertainties of the SRBs, in particular the uncertainties on the SRB side force and yawing moment coefficients. Therefore, one of the main goals of the database update was to reduce the aerodynamic uncertainties for the SRBs especially in the near proximity region. As a result of the uncertainty strategy and model for Version 3 of the database, the uncertainties for the SRB aerodynamic coefficients decreased by $50 \%$ or more from the previous version of the database. Figure 19 shows the uncertainty levels for each aerodynamic coefficient of the RSRB and the contributions of each term to the total level. It also provides the uncertainty levels from the previous version of the database to show the decrease in the important near proximity region of $\Delta X=0-10$ feet. 


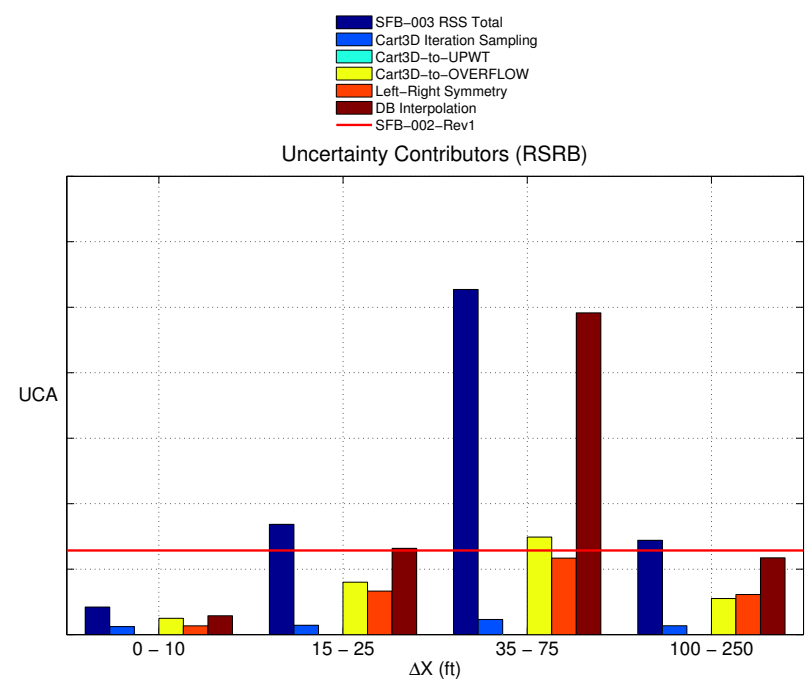

(a) UCA

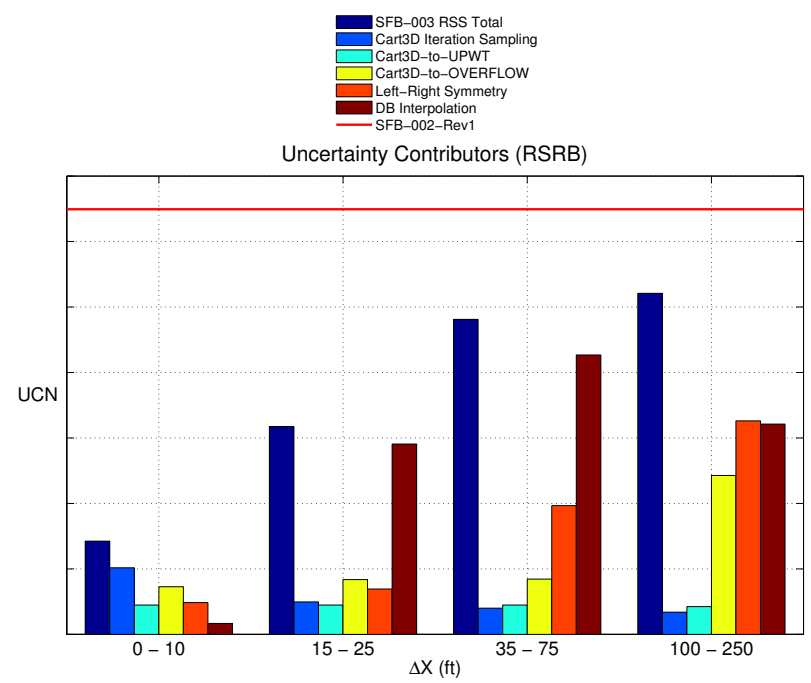

(c) UCN

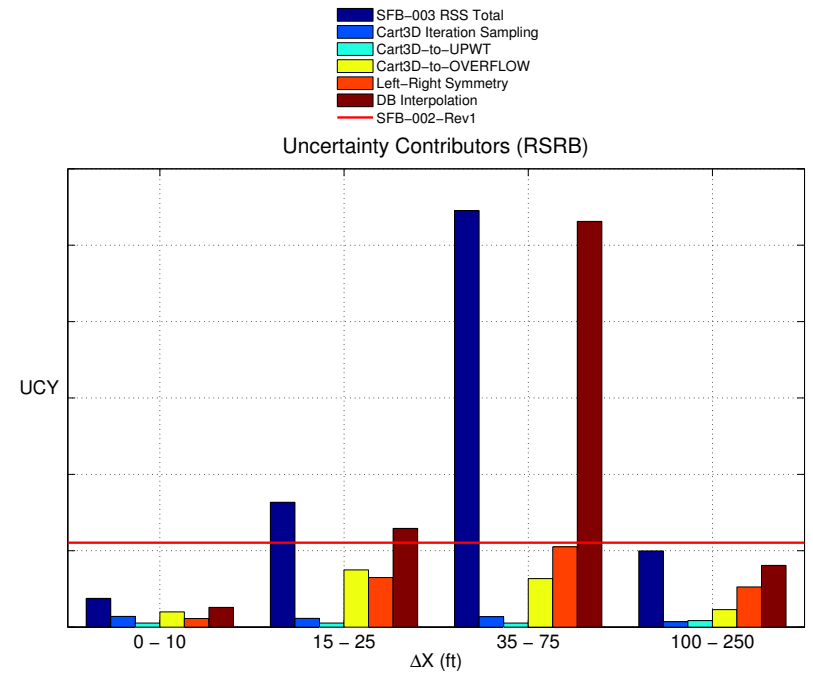

(e) UCY

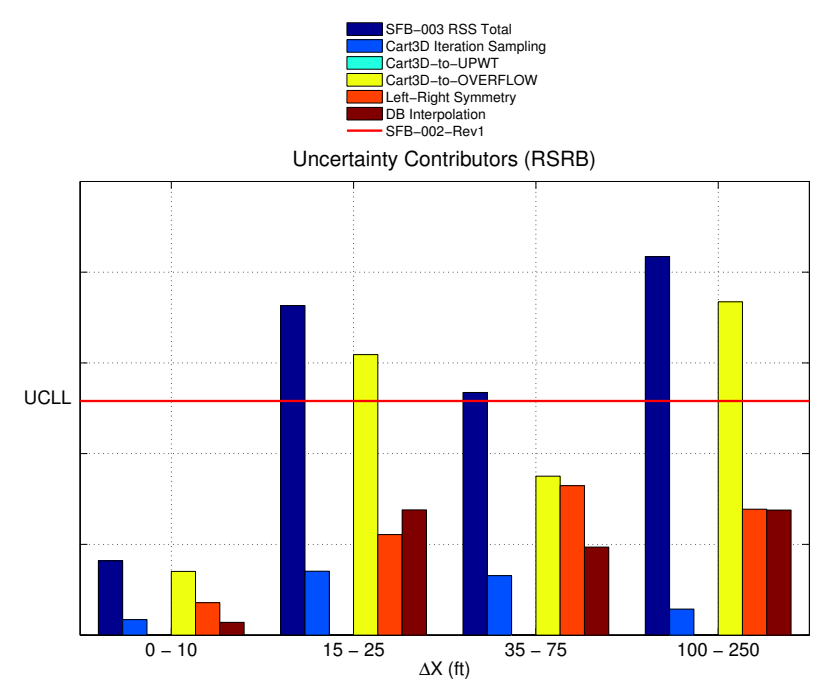

(b) UCLL

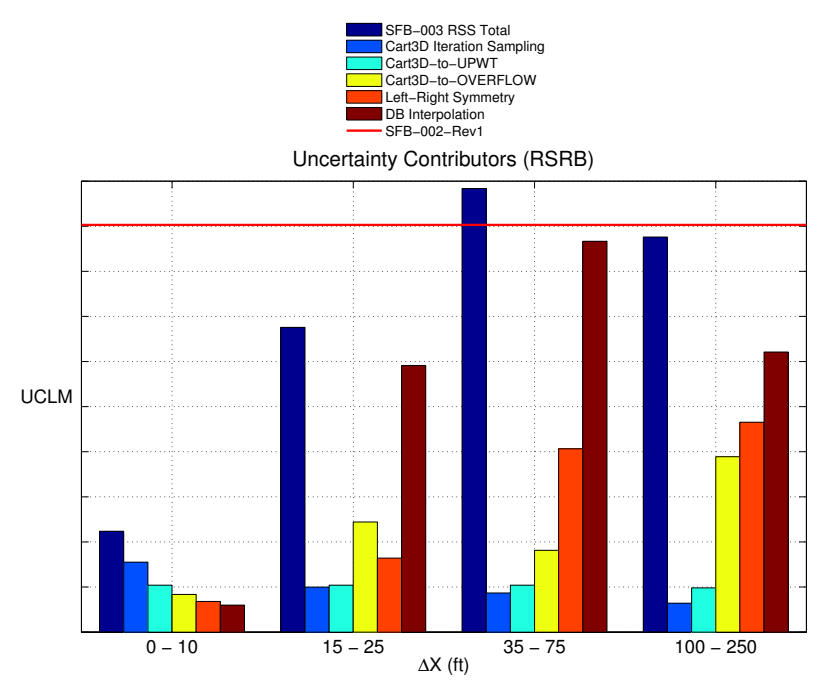

(d) UCLM

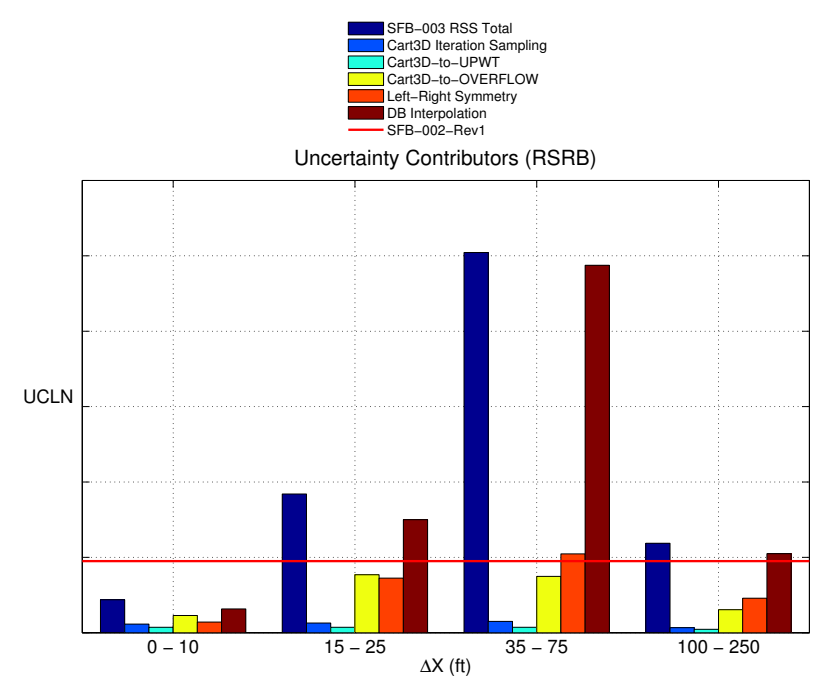

(f) UCLN

Figure 19. Uncertainty levels for the RSRB aerodynamic coefficients with comparison of term contributions and comparison to levels from previous version of database. 


\section{Concluding Remarks}

The development process and uncertainty quantification process for Version 3 of the SLS Booster Separation Aerodynamic F\&M Database was presented in this paper. The booster separation aerodynamic environment is very complicated due to proximity effects between the three separating bodies (core and two SRBs) and jet-induced effects from the twenty-two total engine plumes between the BSMs, SRBs, and CSEs. This aerodynamic environment is very difficult to simulate in a wind tunnel or in CFD simulations. Furthermore, development of the database is complicated by the high dimensionality of the independent variable space since the orientation of the core stage, the location and orientation of the SRBs in relation to the core, the thrust levels of the multiple engines, and the freestream flow conditions are all required information. Simplifying assumptions were made to reduce the number of independent parameters to eight.

The database was developed from a large set of CFD solutions from the Cart3D inviscid flow solver; however, data acquired in a wind tunnel test and data acquired using the OVERFLOW viscous flow solver provided insights into the complex features of the flow and helped to refine the Cart3D grid adaptation methodology to improve the quality of the Cart3D solutions. The Cart3D solution break points were centered around GN\&C trajectory simulations, and a star-shaped pattern was used to choose break points carefully in order to acquire enough data to cover the data space while minimizing time and resource requirements. Response surfaces were generated for the near, mid, and far proximity regions, and these were used to build the final database tables.

The uncertainty model was structured in an attempt to capture the model-form errors, numerical errors, and database modeling errors between the Cart3D-based database and the actual flight environment. Various uncertainty terms including Cart3D-to-WT comparisons, Cart3D-to-OVERFLOW comparisons, Cart3D iteration sampling, database interpolation error, and symmetry assumption errors were used in the uncertainty buildup equation. Most of the terms were analyzed using a statistics-based range analysis methodology that allowed variance trends with $\Delta X$ to be used. The final uncertainty tables were a function of $\Delta X$ to focus on the near, mid, and far proximity regions, with the goal to reduce the uncertainties in the important near proximity region of $\Delta X=0-10$ feet.

The uncertainty levels for the SRB aerodynamic coefficients decreased by $50 \%$ or more from the levels of the previous version of the database. This has resulted in eliminating booster re-contact cases in recent SLS GN\&C simulations and decreased a major risk to the SLS program.

\section{References}

${ }^{1}$ http://www.nasa.gov/sls, "NASA Space Launch System," .

${ }^{2}$ http://people.nas.nasa.gov/ aftosmis/cart3d/, "Cart3D Main Page - NASA Advanced Supercomputing Division," .

${ }^{3}$ http://overflow.larc.nasa.gov/, "NASA OVERFLOW CFD Code," .

${ }^{4}$ Dalle, D. J. and Rogers, S. E., "Output-Based Adaptive Meshing Applied to Space Launch System Booster Separation Analysis," AIAA Paper 2015-3152, AIAA, June 2015.

${ }^{5}$ Dalle, D. J., Rogers, S. E., Chan, W. M., and Lee, H. C., "Inviscid and Viscous CFD Analysis of Booster Separation for the Space Launch System Vehicle," AIAA Paper 2016-xxxx, AIAA, January 2016.

${ }^{6}$ Rogers, S. E., Dalle, D. J., and Chan, W. M., "CFD Simulations of the Space Launch System Ascent Aerodynamics and Booster Separation," AIAA Paper 2015-0778, AIAA, January 2015.

${ }^{7}$ Wilcox, F. J., Pinier, J. T., Chan, D. T., and Crosby, W. A., "Space Launch System Booster Separation Aerodynamic Testing in the NASA Langley Unitary Plan Wind Tunnel," AIAA Paper 2016-xxxx, AIAA, January 2016.

${ }^{8}$ Dalle, D. J., Rogers, S. E., Chan, W. M., and Lee, H. C., "SLS Block 1 SRB Separation Cart3D Documentation," Internal program document, NASA SLS Program, September 2015.

${ }^{9}$ Lophaven, S. N., Nielsen, H. B., and Sondergaard, J., "DACE - A Matlab Kriging Toolbox," Informatics and Mathematical Modeling, Rept. IMM-TR-2002-12, Technical University of Denmark, Aug. 2002.

${ }^{10}$ Sacks, J., Welch, W. J., Mitchell, T. J., and Wynn, H. P., "Design and Analysis of Computer Experiments," Statistical Science, Vol. 4, No. 4, 1989, pp. 409-435.

11 "U.S. Guide to the Expression of Uncertainty in Measurement," Tech. Rep. ANSI/NCSL Z540-2-1997, October 1997.

${ }^{12}$ Wheeler, D. and Chambers, D., Understanding Statistical Process Control, SPC Press, Knoxville, 2 nd ed., 1992.

${ }^{13}$ Wheeler, D., Advanced Topics in Statistical Process Control, SPC Press, Knoxville, 1995.

${ }^{14}$ Wheeler, D., Range Based Analysis of Means, SPC Press, Knoxville, 2003.

${ }^{15}$ Harter, H., "Tables of Range and Studentized Range," The Annals of Mathematical Statistics, Vol. 31, No. 4, December 1960, pp. 1122-1147.

${ }^{16}$ Hemsch, M., Tuttle, D., Houlden, H., and Graham, A., "Measurement of Force Balance Repeatability and Reproducibility in the NTF," AIAA Paper 2004-0771, AIAA, January 2004. 